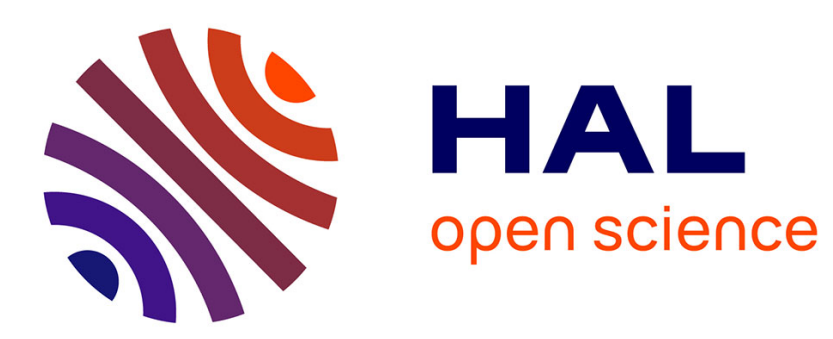

\title{
Efficiency of tidal dissipation in slowly rotating fully convective stars or planets
}

\author{
Jérémie Vidal, Adrian J. Barker
}

\section{To cite this version:}

Jérémie Vidal, Adrian J. Barker. Efficiency of tidal dissipation in slowly rotating fully convective stars or planets. Monthly Notices of the Royal Astronomical Society, 2020, 497 (4), pp.4472-4485. 10.1093/mnras/staa2239 . hal-02907147v2

\section{HAL Id: hal-02907147 \\ https://hal.science/hal-02907147v2}

Submitted on 29 Aug 2020

HAL is a multi-disciplinary open access archive for the deposit and dissemination of scientific research documents, whether they are published or not. The documents may come from teaching and research institutions in France or abroad, or from public or private research centers.
L'archive ouverte pluridisciplinaire HAL, est destinée au dépôt et à la diffusion de documents scientifiques de niveau recherche, publiés ou non, émanant des établissements d'enseignement et de recherche français ou étrangers, des laboratoires publics ou privés. 


\title{
Efficiency of tidal dissipation in slowly rotating fully convective stars or planets
}

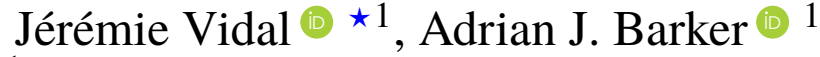 \\ ${ }^{1}$ Department of Applied Mathematics, School of Mathematics, University of Leeds, Leeds, LS2 9JT, UK
}

Accepted 2020 July 23. Received 2020 July 23 ; in original form 2020 July 6

\begin{abstract}
Turbulent convection is thought to act as an effective viscosity in damping equilibrium tidal flows, driving spin and orbital evolution in close convective binary systems. Compared to mixing-length predictions, this viscosity ought to be reduced when the tidal frequency $\left|\omega_{t}\right|$ exceeds the turnover frequency $\omega_{c v}$ of the dominant convective eddies, but the efficiency of this reduction has been disputed. We reexamine this long-standing controversy using direct numerical simulations of an idealized global model. We simulate thermal convection in a full sphere, and externally forced by the equilibrium tidal flow, to measure the effective viscosity $v_{E}$ acting on the tidal flow when $\left|\omega_{t}\right| / \omega_{c v} \gtrsim 1$. We demonstrate that the frequency reduction of $v_{E}$ is correlated with the frequency spectrum of the (unperturbed) convection. For intermediate frequencies below those in the turbulent cascade $\left(\left|\omega_{t}\right| / \omega_{c v} \sim 1-5\right)$, the frequency spectrum displays an anomalous $1 / \omega^{\alpha}$ power law that is responsible for the frequency-reduction $v_{E} \propto 1 /\left|\omega_{t}\right|^{\alpha}$, where $\alpha<1$ depends on the model parameters. We then get $\left|v_{E}\right| \propto 1 /\left|\omega_{t}\right|^{\delta}$ with $\delta>1$ for higher frequencies, and $\delta=2$ is obtained for a Kolmogorov turbulent cascade. A generic $\left|v_{E}\right| \propto 1 /\left|\omega_{t}\right|^{2}$ suppression is next found for higher frequencies within the dissipation range of the convection (but with negative values). Our results indicate that a better knowledge of the frequency spectrum of convection is necessary to accurately predict the efficiency of tidal dissipation in stars and planets resulting from this mechanism.
\end{abstract}

Key words: binaries: close - convection - hydrodynamics - planet-star interactions

\section{INTRODUCTION}

Turbulent convection in stars is believed to dissipate the tidal shear excited by gravitational interactions in close stellar binary or planetary systems, and this process can play an important role in determining the orbital and spin evolution of low-mass binary stars or short-period planets (e.g. Mazeh 2008; Ogilvie 2014). The timescale for these evolutionary processes is inversely proportional to the effective viscosity, and so estimating the stellar (or planetary) viscosity is of crucial importance in applications. The laminar viscosity in convective envelopes is much too small to be relevant for tidal evolution (e.g. in the Sun; Hanasoge \& Sreenivasan 2014), and so turbulent convection is usually thought to act as an effective turbulent viscosity $v_{E}$ that is responsible for damping oscillatory tidal flows. This mechanism is usually invoked to explain the circularization and synchronization of binary systems containing low-mass or solar-like main-sequence stars (e.g. Zahn 1989; Zahn \& Bouchet 1989; Meibom \& Mathieu 2005; Meibom et al. 2006; Van Eylen et al. 2016; Lurie et al. 2017; Triaud et al. 2017; von Boetticher et al. 2019), and evolved stars (e.g. Verbunt \& Phinney 1995; Beck et al. 2018; Price-Whelan \& Goodman 2018).

The effective viscosity due to convection can be estimated by

^ E-mail: vidalje63@gmail.com neglecting the oscillatory nature of the tidal flow such that $v_{E} \simeq v_{c v}$ (leading to the standard constant lag-time tidal model, e.g. Alexander 1973; Eggleton et al. 1998), where $v_{c v}$ is the turbulent viscosity predicted by mixing-length theory (MLT, e.g. Spiegel 1971). Understanding and characterizing the interaction between oscillatory tidal flows and turbulent convection has been referred to as the Achilles' heel of tidal theory (Zahn 2008). Zahn (1966) first realized that $v_{E}$ ought to be reduced when the tidal frequency $\left|\omega_{t}\right|$ is faster than the turnover frequency $\omega_{c v}$ of the dominant convective eddies. The magnitude of this inhibition has been however disputed (e.g. Goodman \& Oh 1997), and two contradictory prescriptions have been used. Zahn $(1966,1989)$ proposed the linear scaling

$v_{E} \propto v_{c v}\left(\left|\omega_{t}\right| / \omega_{c v}\right)^{-1}$,

which is derived by applying MLT arguments assuming that the largest eddies dominate the dissipation, but Goldreich \& Nicholson (1977) proposed instead a quadratic reduction

$v_{E} \propto v_{c v}\left(\left|\omega_{t}\right| / \omega_{c v}\right)^{-2}$

that is derived by assuming that the dominant contribution to the effective viscosity at short tidal periods comes from eddies in the turbulent (Kolmogorov) cascade with a turnover time-scale comparable with the oscillation period.

When equations (1)-(2) are evaluated in stellar models, they 
typically lead to very different predictions for tidal evolutionary time-scales (e.g. Price-Whelan \& Goodman 2018). Thus, application of tidal theory to convection zones remains uncertain, and determining the correct frequency-reduction law of the turbulent viscosity is crucial before we can apply tidal theory to interpret observations of close binaries (e.g. Kirk et al. 2016; Lurie et al. 2017; Van Eylen et al. 2016; Triaud et al. 2017; Price-Whelan \& Goodman 2018) and possibly also short-period planetary orbits (e.g. Rasio et al. 1996). It is possible that the two laws could be valid in different frequency ranges. Indeed, scaling (1) seems to work well when applied to certain stellar oscillations (Gonczi 1982) or in early calculations of pre-main sequence circularization (Zahn \& Bouchet 1989), whereas quadratic scaling (2) could be relevant for much shorter forcing periods, such as those that are relevant for the interaction between acoustic modes and convection (Goldreich \& Keeley 1977; Goldreich et al. 1994; Samadi et al. 2001).

The frequency-reduction law of the turbulent viscosity acting on tidal flows has been also independently revisited with direct numerical simulations (DNS). The two laws were first recovered in separate studies, which support either the linear scaling (Penev et al. 2007, 2009) or the quadratic suppression (Ogilvie \& Lesur 2012; Braviner 2016; Duguid et al. 2020). The coexistence of the two scaling laws has however been found subsequently, using an idealized turbulence model (Goldman 2008) and in our previous global DNS (Vidal \& Barker 2020). These recent results have the potential to reconcile the previous theoretical and numerical findings. Moreover, the recent numerical findings have shed light on the fact that the two scaling laws may be appropriate for different reasons than those originally suggested. On the one hand, the quadratic suppression has been convincingly found for high frequencies $\left|\omega_{t}\right| \gg \omega_{c v}$, particularly those outside the turbulent cascade (Ogilvie \& Lesur 2012; Braviner 2016; Duguid et al. 2020; Vidal \& Barker 2020). On the other hand, the linear reduction, which has been only observed in an intermediate-frequency range (with $\left|\omega_{t}\right| \sim \omega_{c v}$ ), may be correlated with the frequency spectrum of the (unperturbed) convection. Indeed, the convective frequency spectrum is expected to be flatter than the Kolmogorov frequency spectrum in that range, as reported for Boussinesq (Vidal \& Barker 2020) or compressible (e.g. Penev et al. 2011; Horst et al. 2020) convection, such that predictions (1)-(2) may not be generic.

Owing to the importance of this problem to understand tidal evolution, we continue our numerical investigation (Vidal \& Barker 2020) using global DNS of convection in the presence of the equilibrium tidal flow to gain robust physical insights into the efficiency of tidal dissipation in slowly rotating convective stars or planets. Our global model complements the previous local studies in Cartesian geometry (e.g. Ogilvie \& Lesur 2012; Braviner 2016; Duguid et al. 2020), in that we study more realistic tidal flows, and we explore convective flows in stellar-like (or planetary-like) spherical domains in which the flow is free from the influence of artificial periodic (or shearing-periodic) boundary conditions. On the other hand, global DNS are typically more computationally-demanding than local DNS, which prevents us from studying very long tidal periods relative to convective time-scales.

The paper is organized as follows. We present our global model and numerical methods in Section 2, and discuss the general properties of the unperturbed convection in Section 3. Direct computations of the turbulent viscosity are presented in Section 4 . The implications of our results are presented in Section 5, and we conclude the paper in Section 6.

\section{FORMULATION OF THE PROBLEM}

\subsection{Convection model}

We study the interplay between tidal flows and convection using an idealized model of fully convective stars or giant planets. We model a full sphere of radius $R$ and volume $V$, filled with a fluid of uniform (laminar) kinematic viscosity $v$ and thermal diffusivity $\kappa$, and employ spherical coordinates $(r, \theta, \phi)$ centered on the body. The body possibly rotates at the angular velocity $\Omega_{s} \mathbf{1}_{z}$, where $\mathbf{1}_{z}$ is the Cartesian unit vector along the polar axis. We model convection in the Boussinesq approximation (Spiegel 1971), considering slight fluctuations of temperature $\Theta$ and velocity from the motionless conduction state $T_{0}(r)$ sustained by the homogeneous internal heating source $Q_{T}$. The gravitational field is $\boldsymbol{g}=-\gamma \boldsymbol{r}$, where $\boldsymbol{r}$ is the position vector and $\gamma$ is a constant, which represents the leading-order component for a low-mass body that is not very centrally condensed. The primary body is also subjected to tidal forcing from an orbiting companion, which drives large-scale tidal flows in the fluid interior (Ogilvie 2014; Le Bars et al. 2015). Following Goodman \& Oh (1997), we divide the total velocity field $\boldsymbol{u}+\boldsymbol{U}_{0}$ into two components, a turbulent convective flow $\boldsymbol{u}$ and a background large-scale tidal flow $\boldsymbol{U}_{0}$ (see below).

We employ dimensionless quantities for the simulations, adopting $R$ as the length scale, the viscous time-scale $R^{2} / v$ as the timescale, and $\left(v Q_{T} R^{2}\right) /\left(6 \kappa^{2}\right)$ as the unit of temperature (as in Vidal $\&$ Barker 2020). The dimensionless Boussinesq equations for the fluctuations $[\boldsymbol{u}, \Theta]$ in the rotating frame are

$$
\begin{aligned}
\frac{\partial \boldsymbol{u}}{\partial t}+(\boldsymbol{u} \cdot \boldsymbol{\nabla}) \boldsymbol{u} & =-\boldsymbol{\nabla} p+\boldsymbol{\nabla}^{2} \boldsymbol{u}+\operatorname{Ra} \Theta \boldsymbol{r}-\boldsymbol{f}, \\
\frac{\partial \Theta}{\partial t}+(\boldsymbol{u} \cdot \boldsymbol{\nabla}) \Theta & =\frac{1}{P r}\left[2 \boldsymbol{u} \cdot \boldsymbol{r}+\nabla^{2} \Theta\right]-Q, \\
\boldsymbol{\nabla} \cdot \boldsymbol{u} & =0,
\end{aligned}
$$

with the dimensionless (reduced) pressure $p$ and

$\boldsymbol{f}=(2 / E) \mathbf{1}_{z} \times \boldsymbol{u}+(\boldsymbol{u} \cdot \boldsymbol{\nabla}) \boldsymbol{U}_{0}+\left(\boldsymbol{U}_{0} \cdot \boldsymbol{\nabla}\right) \boldsymbol{u}$,

$\boldsymbol{Q}=\left(\boldsymbol{U}_{0} \cdot \boldsymbol{\nabla}\right) \Theta$.

We have discarded the term $\left(\boldsymbol{U}_{0} \cdot \boldsymbol{\nabla}\right) T_{0}$ in the temperature equation, since it should be negligible when $\beta \ll 1$ (e.g. Lai et al. 1993, in the ellipsoidal geometry). We have also introduced in equations (3) the Rayleigh number $R a$, the Prandtl number $P r$ and the Ekman number $E$. They are given by

$R a=\frac{\alpha_{T} \gamma Q_{T} R^{6}}{6 v \kappa^{2}}, \quad \operatorname{Pr}=\frac{v}{\kappa}, \quad E=\frac{v}{\Omega_{s} R^{2}}$,

where $\alpha_{T}$ is the thermal expansion coefficient. The Rayleigh number measures the strength of the convective driving, and the Ekman number the strength of viscous diffusion with respect to global rotation. Since many low-mass stars are slow rotators (e.g. Nielsen et al. 2013; Newton et al. 2018), we will mainly ignore global rotation in the DNS by setting $E=+\infty$ (though we will also consider a few slowly rotating cases, see below).

Equations (3) are complemented with boundary conditions at the (dimensionless) spherical boundary $r=1$. For the temperature, we employ the isothermal condition $\Theta=0$ (we expect to obtain similar results using fixed flux conditions). To avoid spurious numerical issues associated with angular momentum conservation in global simulations of tidal flows (e.g. as observed in Favier et al. 2014), we enforce the no-slip (NS) boundary conditions (BC) $\boldsymbol{u}=\mathbf{0}$. The latter BC does not qualitatively affect the (small-scale) turbulent flows driven in the bulk in our simulations, compared to the more realistic stress-free (or free-surface) BC for stellar applications. 


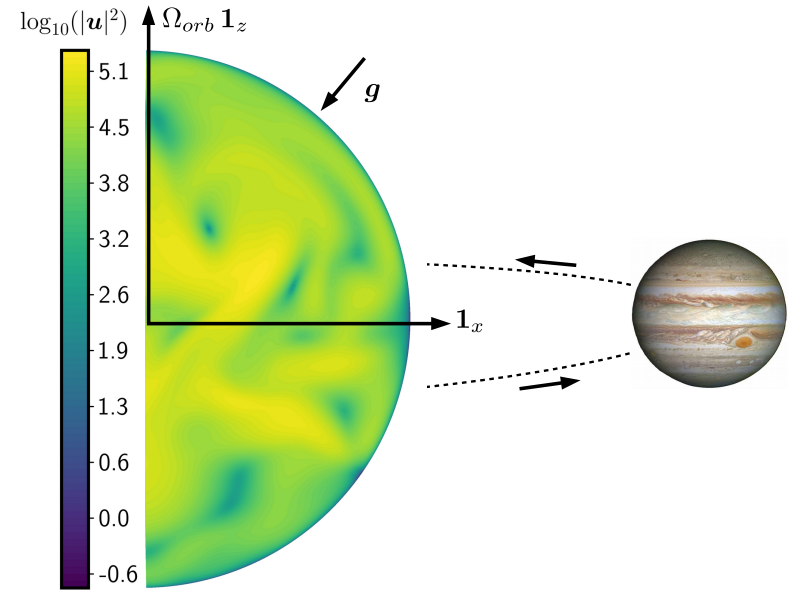

Figure 1. Sketch of the tidal problem in the inertial frame. The companion is orbiting around the fluid body in the orbital plane (dashed line), with angular velocity $\Omega_{\text {or } b} \mathbf{1}_{z}$. Color bar illustrates $\log _{10}\left(|\boldsymbol{u}|^{2}\right)$ for our DNS with $R a=8 \times 10^{6}$.

\subsection{Tidal forcing}

Previous numerical studies modeled the tidal flow with either an (ad-hoc) external forcing (Penev et al. 2009), or with a background unidirectional shear flow in a shearing box (Ogilvie \& Lesur 2012; Braviner 2016; Duguid et al. 2020). For a more realistic astrophysical model, we consider self-consistently the large-scale (nonwavelike) equilibrium tidal flow in a homogeneous body. We assume that the companion is a point mass, moving on an aligned circular orbit around the star with the angular velocity $\Omega_{\text {orb }} \mathbf{1}_{z}$ (as depicted in Fig. 1). Thus, the dominant component of the tidal potential has the spherical harmonic degree $l=2$ and azimuthal order $m=2$ (Ogilvie 2014). In the frame rotating with the fluid at the rate $\Omega_{s}$, the resulting (dimensionless) flow is in the $x y$-plane and takes the form (e.g. Barker \& Lithwick 2013)

$\boldsymbol{U}_{0}=-\frac{\omega_{t} \beta}{2}\left(\begin{array}{cc}\sin \left(\omega_{t} t\right) & \cos \left(\omega_{t} t\right) \\ \cos \left(\omega_{t} t\right) & -\sin \left(\omega_{t} t\right)\end{array}\right)\left(\begin{array}{l}x \\ y\end{array}\right)$,

where $\beta \ll 1$ is the dimensionless tidal amplitude (roughly the ratio of tidal displacement to unperturbed radius $), \omega_{t}=2\left(E^{-1}-E_{\text {orb }}^{-1}\right)$ is the dimensionless forcing frequency and $E_{\text {orb }}^{-1}=\left(\Omega_{\text {orb }} R^{2}\right) / v$ is the dimensionless orbital frequency.

\subsection{Numerical modeling}

We follow the numerical implementation introduced in Vidal \& Barker (2020) to account for tidal flows. The non-linear equations (3) are solved in their weak variational form by using the spectralelement code Nek5000 (e.g. Fischer et al. 2007). The computational domain is decomposed into 3584 non-overlapping hexahedral elements. Within each element, the velocity (and pressure) is represented as Lagrange polynomials of order $\mathcal{N}$ (respectively, $\mathcal{N}-2$ ) on the Gauss-Lobatto-Legendre (Gauss-Legendre) points. Temporal discretization is accomplished by a third-order method, based on an adaptive and semi-implicit scheme in which the non-linear and Coriolis terms are treated explicitly, and the remaining linear terms are treated implicitly. Solutions are de-aliased following the $3 / 2$ rule, such that $3 \mathcal{N} / 2$ grid points are used in each dimension for the non-linear terms, whereas only $\mathcal{N}$ points are used for the linear terms. We have checked the numerical accuracy in targeted simulations by varying the polynomial order from $\mathcal{N}=7$ to $\mathcal{N}=9$.
Table 1. Characteristics of (unperturbed) DNS with NS conditions. Rayleigh number $R a$, Prandtl number $P r$, convective velocity $u_{c v}$, and turbulent length scale $l_{E}$.

\begin{tabular}{lccc}
\hline$R a, P r$ & $E$ & $u_{c v}$ & $l_{E}$ \\
\hline $1 \times 10^{5}, 1.0$ & $+\infty$ & $(1.84 \pm 0.1) \times 10^{1}$ & $(4.6 \pm 0.8) \times 10^{-1}$ \\
$3 \times 10^{5}, 1.0$ & $+\infty$ & $(3.18 \pm 0.2) \times 10^{1}$ & $(3.9 \pm 1.1) \times 10^{-1}$ \\
$6 \times 10^{5}, 1.0$ & $+\infty$ & $(4.40 \pm 0.2) \times 10^{1}$ & $(3.5 \pm 1.1) \times 10^{-1}$ \\
$1 \times 10^{6}, 1.0$ & $+\infty$ & $(5.64 \pm 0.3) \times 10^{1}$ & $(3.2 \pm 1.0) \times 10^{-1}$ \\
$2 \times 10^{6}, 1.0$ & $+\infty$ & $(7.74 \pm 0.4) \times 10^{1}$ & $(2.9 \pm 1.0) \times 10^{-1}$ \\
$4 \times 10^{6}, 1.0$ & $+\infty$ & $(1.00 \pm 0.1) \times 10^{2}$ & $(2.5 \pm 0.8) \times 10^{-1}$ \\
$8 \times 10^{6}, 1.0$ & $+\infty$ & $(1.31 \pm 0.1) \times 10^{2}$ & $(2.2 \pm 0.2) \times 10^{-1}$ \\
$1 \times 10^{6}, 0.3$ & $+\infty$ & $(1.43 \pm 0.1) \times 10^{2}$ & $(2.5 \pm 0.9) \times 10^{-1}$ \\
$1 \times 10^{6}, 1.0$ & $10^{-1}$ & $(5.64 \pm 0.3) \times 10^{1}$ & $(3.1 \pm 1.0) \times 10^{-1}$ \\
$1 \times 10^{6}, 1.0$ & $10^{-2}$ & $(4.86 \pm 0.3) \times 10^{1}$ & $(2.9 \pm 1.0) \times 10^{-1}$ \\
\hline
\end{tabular}

The efficiency of tidal dissipation is investigated by computing an effective volume-averaged viscosity coefficient $v_{E}$, introducing the volume average $\langle\cdot\rangle_{V}=(1 / V) \int_{V} \cdot \mathrm{d} V$. The forcing amplitude $\beta$ must be large enough to obtain a measurable tidal response, but too large values could strongly modify the results when the amplitude of the tidal flow is much larger than the convective flow (e.g. see in Penev et al. 2009; Duguid et al. 2020). Only small differences in the properties of the convection have been found for the values of $\beta$ considered below (always smaller than a few percent for the volume-averaged quantities when $\beta \leq 5 \times 10^{-2}$, not shown).

Finally, we initiated the convection with random noise to the temperature field and let it saturate without tides (i.e. $\beta=0$ ) for most of the simulations, before switching on the equilibrium tidal flow. We have checked that initiating the convection together with the tidal flow does not lead to noticeably different results.

\section{UNPERTURBED CONVECTION}

We simulate highly super-critical convection with $R a \gg R a_{c}$ and $\operatorname{Pr}=1$, where the critical value for linear onset, computed using a dedicated linear solver (Vidal \& Schaeffer 2015; Monville et al. 2019), is $R a_{c}=4019$ with NS conditions (the latter value corrects the onset given in Vidal \& Barker 2020, which corresponds instead with the critical value for stress-free BC). The parameters and outputs for the DNS with $\beta=0$ are summarized in table 1 . The spatial spectrum of the unperturbed convection is illustrated in Fig. 2 . The spectra are well converged with our adopted resolution and they exhibit (small) inertial-like ranges, with a Kolmogorov scaling $(\propto-5 / 3)$ that emerges more clearly when $R a$ is increased.

For astrophysical applications, the convection is often characterized using MLT by the (unperturbed) turbulent viscosity $v_{c v} \sim u_{c v} l_{E}$, with a typical amplitude of the flow $u_{c v}$ and a typical length scale of the turbulent eddies $l_{E}$. To define the convective velocity $u_{c v}$, we use the volume-averaged root-mean-square radial velocity $u_{c v}=\left(\left\langle u_{r}^{2}\right\rangle_{V}\right)^{1 / 2}$ that characterizes the radial mixing. We find $u_{c v} \propto R a^{0.45}$ in the DNS (top panel in Fig. 3), which is in reasonably good agreement with the MLT scaling $\propto R a^{1 / 2}$ expected in the fully turbulent regime (e.g. Spiegel 1971). This indicates that the convective velocities in our DNS are in an approximately diffusion-free regime, as is expected in stars and planets.

The length scale $l_{E}$ is usually defined as a function of the local pressure scale height in stellar interiors, but this definition cannot be self-consistently employed in Boussinesq models. Estimating $l_{E}$ in global models is difficult (except for rapidly rotating convection, 


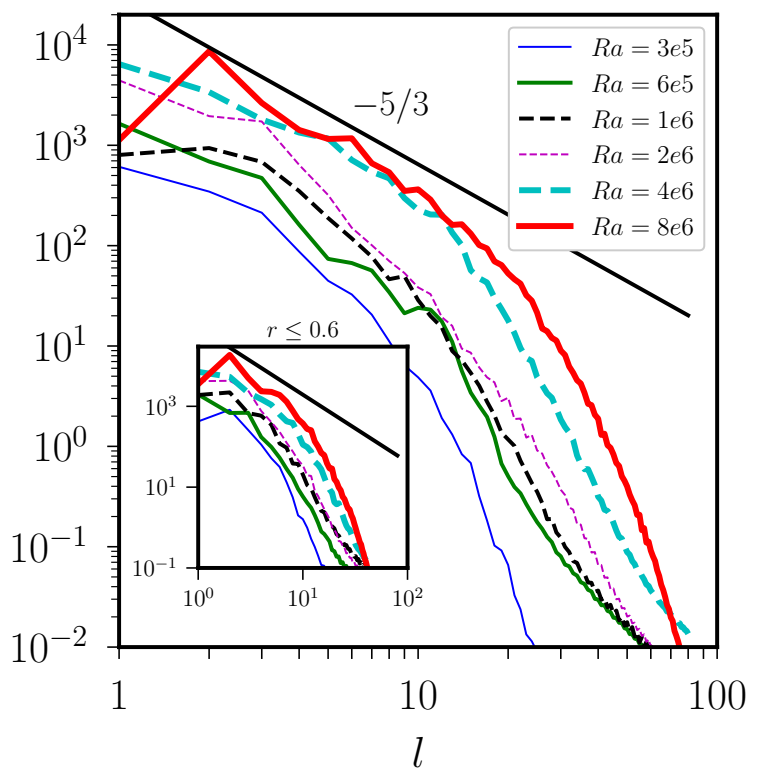

Figure 2. Instantaneous volume-averaged spectrum of the kinetic energy, as a function of the spherical harmonic degree $l \geq 1$ (using orthonormalized spherical harmonics). Thick black line shows the Kolmogorov power law $l^{-5 / 3}$. Spectra have been computed by interpolating the data to a spherical grid, and then by performing a spherical harmonics analysis (Schaeffer 2013). Inset shows the volume-averaged spectra restricted to $r \leq 0$. 6 .
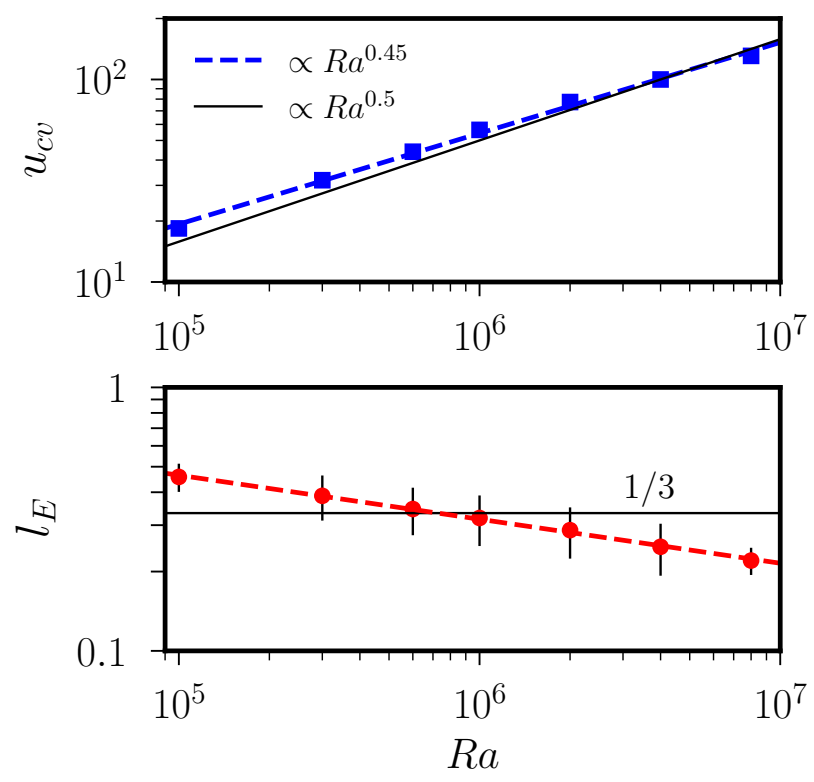

Figure 3. Top panel: Convective velocity $u_{c v}$ as a function of $R a$ in DNS. Bottom panel: Length scale $l_{E}$ as a function of $R a$. Dashed line is the power law $l_{E}=3.17 R a^{-0.17}$. Horizontal line indicates the value $l_{E}=1 / 3$ considered in Vidal \& Barker (2020) with $R a=10^{6}$.

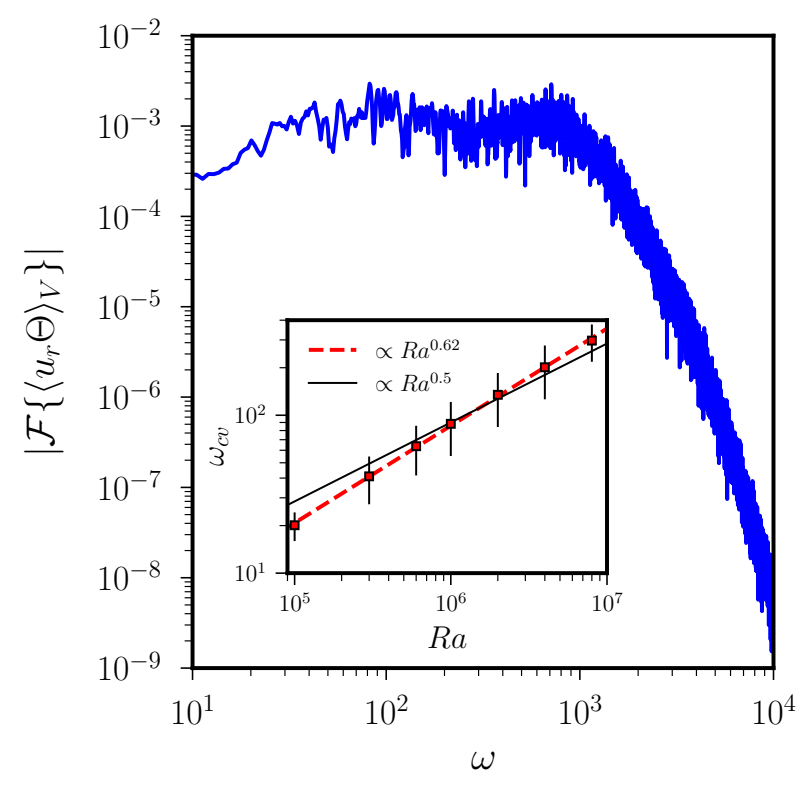

Figure 4. Frequency spectrum of the convective flux $\left\langle u_{r} \Theta\right\rangle_{V}$ in DNS with $R a=4 \times 10^{6}$. Inset panel shows the convective (angular) frequency $\omega_{c v}$ as a function of $R a$.

as reported in Guervilly et al. 2019), but a useful characterization of turbulent flows is the Taylor wavenumber $k_{T}$ (e.g. Rieutord 2014)

$k_{T}=\sqrt{\left\langle|\boldsymbol{\nabla} \times \boldsymbol{u}|^{2}\right\rangle_{V} /\left\langle|\boldsymbol{u}|^{2}\right\rangle_{V}}$,

from which we can estimate a turbulent length scale as $l_{E}=\pi / k_{T}$ (based on the half wavelength). Note that this scale does not represent the energetically-dominant eddies, but a scale intermediate between the "outer scale" and the dissipation scales, and fairly represents the mean size of the eddies in the turbulent cascade. Indeed, in our DNS that do not possess very long inertial ranges, $l_{E}$ works reasonably well to define the typical size of the turbulent eddies (which we have verified by visual inspection of the flow). We show in Fig. 3 (bottom panel) the evolution of $l_{E}$ as a function of $R a$ in the DNS, and observe that the length scale displays the power law ${ }^{1}$ $l_{E} \propto R a^{-0.17}$. It also agrees with the value $l_{E} \simeq 1 / 3$ at $R a=10^{6}$, which was considered in Vidal \& Barker (2020).

An estimate of the the convective (angular) frequency $\omega_{c v}$ is also required. By analogy with stellar models, one can define the convective frequency based on the input parameters (as also considered in Ogilvie \& Lesur 2012). To do so, we introduce the dimensionless Brunt-Väisälä frequency $N(r)$ given here by $N^{2}(r)=$ $-2 r^{2} R a / P r$, and define a typical convective frequency $\omega_{c v} \sim\left|N_{0}\right|$ with the mean radial value $\left|N_{0}\right|=\left|N^{2}(1)\right|^{1 / 2} / 2 \propto(R a / P r)^{1 / 2}$, whose scaling agrees with MLT (e.g. Spiegel 1971). Alternatively, a more accurate definition could be based on the turbulent properties of the convective flows. In the following, we compute the frequency spectrum of the time series $X(t)$ defined as $|\mathcal{F}\{X(t)\}|$,

1 A similar scaling for $l_{E}$ can be obtained by considering that it should scale like the geometric mean $l_{E} \sim(\eta R)^{0.5}$, with the outer scale $R \sim 1$ and the dissipation scale $\eta \sim R / R e^{3 / 4}$ (Rieutord 2014), where $R e$ is a Reynolds number of the large-scale eddies (assuming $R e \sim R a^{0.5}$, consistently with Fig. 3). This gives $l_{E} \sim R a^{-0.19} R$ in dimensional units. 


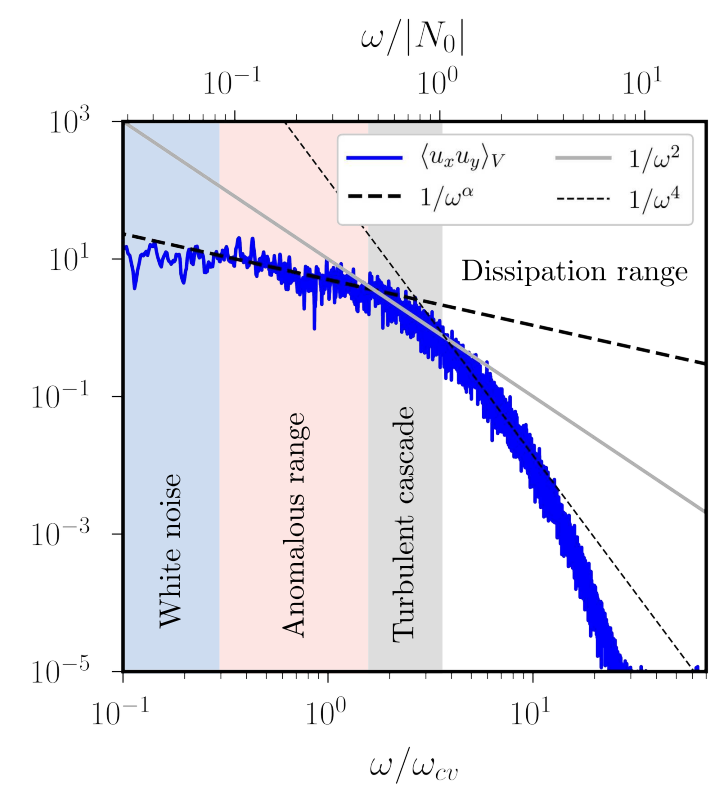

Figure 5. Frequency spectrum of $\left\langle u_{x} u_{y}\right\rangle_{V}$ for DNS with $R a=4 \times 10^{6}$. The thick dashed line shows the power law $1 / \omega^{0.66}$, and the thick gray line indicates the scaling $1 / \omega^{2}$ expected for a Kolmogorov cascade.

where $\mathcal{F}$ is the Discrete Fourier Transform, as a function of the angular frequency $\omega$. We first remove the mean value of the time series and then apply a Hanning window function before we compute numerically the Fourier transform (using the FFT algorithm, and normalizing by the length of the signal). One may define $\omega_{c v}$ as the frequency that provides the maximum contribution to the convective flux $\left\langle u_{r} \Theta\right\rangle_{V}$, but the convective frequency is actually poorly constrained from the spectrum of this quantity, which does not exhibit a clearly defined peak (see Fig. 4). We choose to instead define the convective frequency as $\omega_{c v}=u_{E} / l_{E}$, to be consistent with simple MLT expectations. We find that $\omega_{c v} \propto R a^{0.62}$ in the DNS (see inset), which is quite close to the MLT prediction $\omega_{c v} \propto R a^{0.5}$ (e.g. Spiegel 1971).

We show in Fig. 5 the frequency spectrum of the Reynolds stress component $\left\langle u_{x} u_{y}\right\rangle_{V}$, where the angular frequencies have been normalized by $\omega_{c v}$ (bottom axis) and $\left|N_{0}\right|$ (top axis), for the illustrative DNS with $R a=4 \times 10^{6}$. Several different regimes are observed (which are also relevant for the spectrum of the kinetic energy, not shown). For very low frequencies $\omega_{c v} \lesssim O\left(10^{-1}\right)$, we observe frequency-independent white noise. Within an intermediate frequency range (here $10^{-1} \lesssim \omega / \omega_{c v} \leq O(1)$ ), denoted below as the anomalous range, the spectrum is characterized by an anomalous $1 / \omega^{\alpha}$ power law with exponents $\alpha<1$ that vary with $R a$ and $P r$ in full spheres (as we will discuss further below). For larger frequencies $\omega / \omega_{c} \geq O(1)$ in the turbulent cascade, the spectrum first displays the power law $1 / \omega^{2}$ expected for Kolmogorov turbulence (Landau \& Lifshitz 1987; Kumar \& Verma 2018). Finally, the frequencies belong to the dissipation range of the convection when $\omega / \omega_{c} \gg 1$, first with the power-law scaling $1 / \omega^{4}$ in a narrow frequency interval (as found in laboratory experiments, see in Liot et al. 2016) and then with a steeper decay.

\section{EFFICIENCY OF TIDAL DISSIPATION}

\subsection{Effective viscosity coefficient}

We primarily extract the turbulent viscosity from our DNS by defining an effective viscosity coefficient $v_{E}$, which is computed by balancing the mean rate at which convection does work on the tidal flow with the mean rate of viscous dissipation of the latter flow (e.g. Goodman \& Oh 1997; Duguid et al. 2020; Vidal \& Barker 2020). This leads to $v_{E}=\left\langle v_{t}(r, \theta, \phi)\right\rangle_{V}$ with

$v_{t}(r, \theta, \phi)=-\frac{1}{\left(\omega_{t} \beta\right)^{2} \Delta T} \int_{t_{0}}^{T} \boldsymbol{u} \cdot\left[(\boldsymbol{u} \cdot \boldsymbol{\nabla}) \boldsymbol{U}_{0}\right] \mathrm{d} t$

and the integrand

$$
\begin{aligned}
\boldsymbol{u} \cdot\left[(\boldsymbol{u} \cdot \boldsymbol{\nabla}) \boldsymbol{U}_{0}\right]=-\frac{\omega_{t} \beta}{2}\left[\left(u_{x}^{2}-u_{y}^{2}\right) \sin \left(\omega_{t} t\right)\right. & \\
& \left.+2 u_{x} u_{y} \cos \left(\omega_{t} t\right)\right],
\end{aligned}
$$

where $\Delta T=T-t_{0}$ is the time-interval used for integration (with $t_{0}$ being an appropriate initial time in the saturated regime). The time average in expression (8a) is obtained by fitting a linear slope to the cumulative time integral to reduce turbulent noise. (e.g. see fig. 13 in Duguid et al. 2020). Global simulations in the presence of large-scale tidal flows are very demanding, because they must be run for a sufficiently long duration to reduce noise. We have therefore integrated each simulation with a tidal flow for at least one viscous time unit (i.e. $\Delta T \geq 1$ ), corresponding with more than a hundred tidal periods, to obtain converged statistics for the effective viscosity. Finally, since the background flow strictly does not satisfy the boundary conditions in a sphere, we have verified that the volume average is not dominated by regions near the boundary, and is instead due to interactions with turbulent flows in the bulk (not shown here, but see fig. 5 in Vidal \& Barker 2020).

We show in Fig. 6 the direct computations of $v_{E}$ in the DNS with $R a=10^{6}$ and $R a=4 \times 10^{6}$, assuming a tidal amplitude of $\beta=5 \times 10^{-2}$ (which is e.g. a relevant value for a solar-mass binary in a one-day orbit). We also over-plot the frequency spectrum of the Reynolds stress component $\mathcal{F}\left\{\left\langle u_{x} u_{y}\right\rangle_{V}\right\}$ as the gray lines in both panels. The clearest result evident in Fig. 6 is that $v_{E}$ decreases as the ratio $\left|\omega_{t}\right| / \omega_{c v}$ is increased, which means that the efficiency of the dissipation is reduced for fast tides. For the particular DNS with $R a=10^{6}$, the two canonical frequency-reduction laws (linear and quadratic) are approximately obtained, which were previously discussed in Vidal \& Barker (2020). However, our more thorough analysis reveals that the frequency-reduction law follows several successive power laws that are in good agreement with the frequency spectrum of the unperturbed convection. Within the anomalous (intermediate-frequency) range where the frequency spectrum of the unperturbed convection varies as $1 / \omega^{\alpha}$ (with power exponents $\alpha<1$ in full spheres), the viscosity is reduced as $v_{E} \propto 1 /\left(\left|\omega_{t}\right| / \omega_{c v}\right)^{\alpha}$. Then, for higher frequencies in the (narrow) turbulent cascade that displays the Kolmogorov power law $1 / \omega^{2}$, the previous frequency-reduction scaling ceases to be valid and is replaced by a quadratic reduction for the effective viscosity (with only positive values).

Therefore, we obtain two successive frequency-reduction laws for the effective viscosity for frequencies below the dissipation range of the turbulence. Although our turbulent cascade corresponds here to a narrow frequency interval, our results confirm that a Kolmogorov spectrum is associated with a quadratic reduction (as postulated by Goldreich \& Nicholson 1977, though it is unclear whether their mechanism applies in detail). However, for smaller 

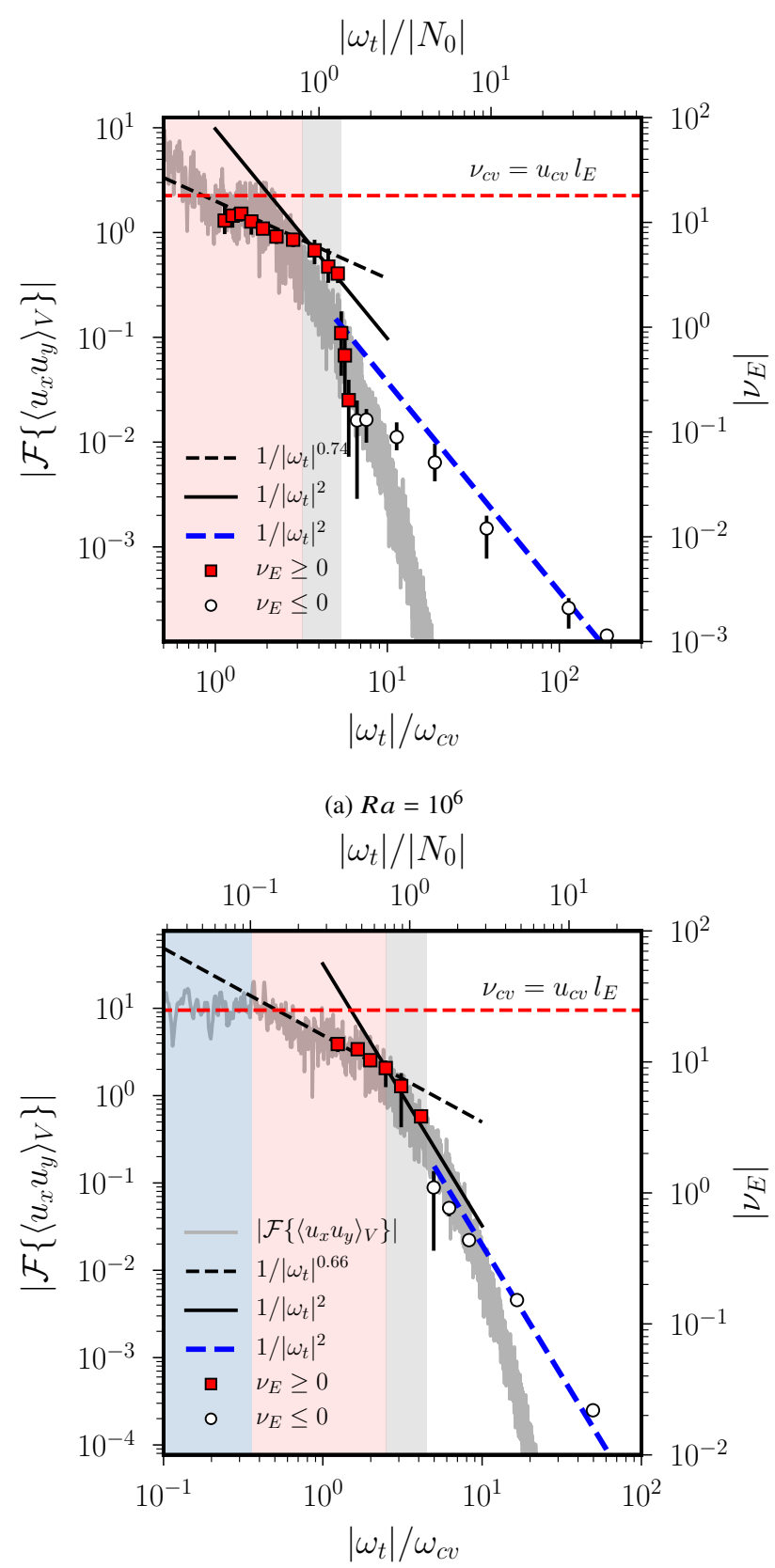

(b) $R a=4 \times 10^{6}$

Figure 6. Direct measurements of the effective viscosity $v_{E}$ in non-rotating DNS with $P r=1$ and $\beta=5 \times 10^{-2}$, as a function of $\left|\omega_{t}\right| / \omega_{c v}$ or $\left|\omega_{t}\right| /\left|N_{0}\right|$. Squares: $v_{E}>0$. Circles: $v_{E}<0$. Horizontal dashed lines: MLT expectation $v_{c v}=u_{c v} l_{E}$ in the low-frequency regime (i.e. $\left.\left|\omega_{t}\right| \ll \omega_{c v}\right)$. The gray curve shows the frequency spectrum of $\left\langle u_{x} u_{y}\right\rangle_{V}$ for unperturbed convection with $\beta=0$, as a function of the scaled angular frequency $|\omega| / \omega_{c v}$ (same horizontal values as $\left|\omega_{t}\right| / \omega_{c v}$ ). Background colors refer to figure 5 .

frequencies in the anomalous range (i.e. outside the turbulent cascade), the frequency reduction of the effective viscosity is neither quadratic nor linear, but follows instead the anomalous frequency spectrum $1 / \omega^{\alpha}$ of the convection. Moreover, the power exponent $\alpha<1$ is reduced in full spheres when the Rayleigh number is increased, for instance with $\alpha=0.74 \pm 0.05$ when $R a=10^{6}$ and $\alpha=0.66 \pm 0.05$ when $R a=4 \times 10^{6}$.

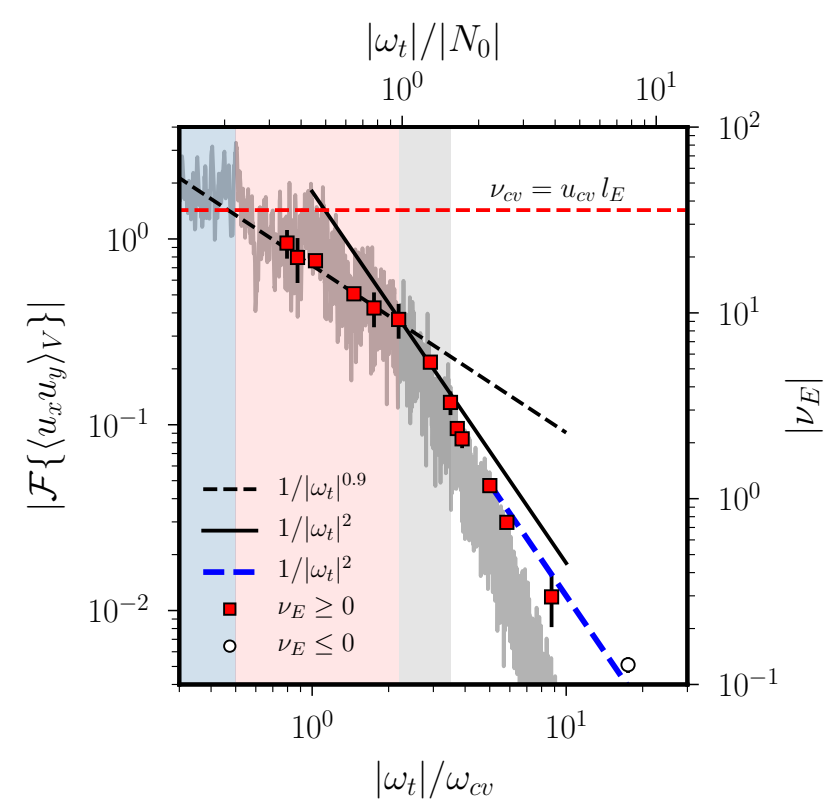

Figure 7. Direct measurements of the effective viscosity $v_{E}$ in non-rotating DNS as a function of $\left|\omega_{t}\right| / \omega_{c v}$ (or $\left|\omega_{t}\right| /\left|N_{0}\right|$ ). DNS with $R a=10^{6}$, $P r=0.3$ and $\beta=5 \times 10^{-2}$. Squares: $v_{E}>0$. Circles: $v_{E}<0$. Horizontal dashed lines: MLT prediction $v_{c v}=u_{c v} l_{E}$. The gray curve shows the frequency spectrum of $\left\langle u_{x} u_{y}\right\rangle_{V}$ for unperturbed convection with $\beta=0$, as a function of the scaled angular frequency $|\omega| / \omega_{c v}$ (same horizontal values as $\left.\left|\omega_{t}\right| / \omega_{c v}\right)$. Background colors refer to Fig. 5 .

We show in Fig. 7 the effective viscosity measured in DNS with $R a=10^{6}$ and $P r=0.3$ (this is relevant for liquid metals, e.g. Kaplan et al. 2017). Exploring cases with smaller $\operatorname{Pr}$ is important because $\mathrm{Pr}$ in stellar or planetary convection zones is much smaller than unity (e.g. Hanasoge \& Sreenivasan 2014). The frequency range of the Kolmogorov cascade is slightly larger in this case compared to Fig. 6, and more importantly the transition between positive and negative values occurs at larger tidal frequencies (within the dissipation range) but always when $\left|v_{E}\right| \lesssim v$. Note also that the value of the exponent $\alpha$ is different in the intermediate-frequency regime, showing that $\alpha$ also depends on $\operatorname{Pr}$, which indicates a parameter-dependence within the anomalous range.

Note that we have been unable to accurately determine $v_{E}$ in the low-frequency regime $\left(\left|\omega_{t}\right| \lesssim \omega_{c v}\right)$ with these simulations. This is because the amplitude of the tidal flow in this regime was too weak to give a sufficiently strong signal-to-noise ratio. A crude extrapolation of our results into the low frequency regime is broadly consistent with expectations from MLT though, which would predict $v_{E} \propto v_{c v} \sim u_{c v} l_{E}$ when $\left|\omega_{t}\right| \rightarrow 0$. The proportionality constant is often assumed to be $1 / 3$ without rigorous justification (e.g Zahn 1989; Ogilvie \& Lin 2007), based on the analogy with kinetic theory for a microscopic viscosity. Here we instead find values close to 1 , or in fact in excess of 1 if $v_{E}$ continues to follow the spectrum for smaller $\left|\omega_{t}\right| / \omega_{c v}$, indicating more efficient dissipation at low frequencies from this mechanism than the naive application of MLT would predict. This result is broadly consistent with local simulations (Duguid et al. 2020), and prior theoretical work obtained with an idealized turbulence model (e.g. Goldman 2008). 


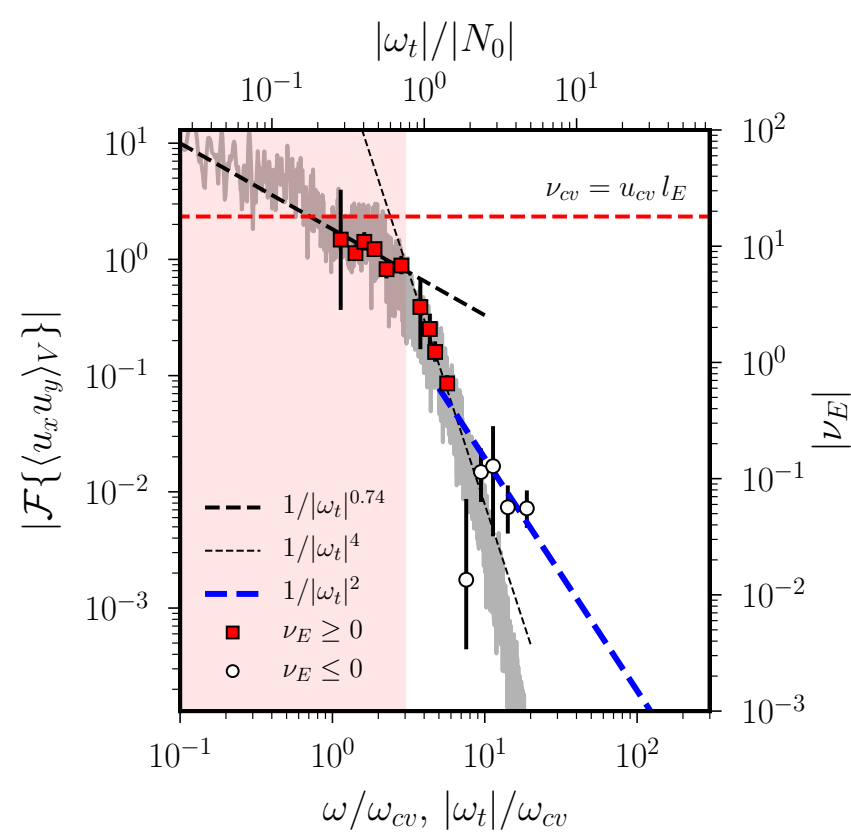

Figure 8. Direct measurements of the effective viscosity $v_{E}$ in non-rotating DNS as a function of $\left|\omega_{t}\right| / \omega_{c v}$ (or $\left|\omega_{t}\right| /\left|N_{0}\right|$ ), showing the transition to negative values of $\nu_{E}$. DNS with $R a=10^{6}, \operatorname{Pr}=1$ and $\left|\omega_{t}\right| \beta=$ 20. Squares: $v_{E}>0$. Circles: $v_{E}<0$. Horizontal dashed lines: MLT prediction $v_{c v}=u_{c v} l_{E}$. The gray curve shows the frequency spectrum of $\left\langle u_{x} u_{y}\right\rangle_{V}$ for unperturbed convection with $\beta=0$, as a function of the scaled angular frequency $|\omega| / \omega_{c v}$ (same horizontal axis as $\left|\omega_{t}\right| / \omega_{c v}$ ). Background colors refer to Fig. 5.

\subsection{Negative values}

Statistically significant negative values of the turbulent viscosity are found in Figs 6 and 7 for much higher frequencies within the dissipation range, which are consistent with previous local results and asymptotic theory (Ogilvie \& Lesur 2012; Duguid et al. 2020). The transition towards negative values is better illustrated in Fig. 8 using DNS with $R a=10^{6}$ and $P r=1$, but with the fixed amplitude $\left|\omega_{t}\right| \beta=20$ for the tidal flow (instead of fixing $\beta$ ). This allows us to investigate more efficiently the transition between positive and negative values, without disturbing (to the same extent) the frequency spectrum of the convection contrary to Figs 6-7 (for which the amplitude of the tidal flow increases when $\left|\omega_{t}\right|$ increases, see fig. 3 in Vidal \& Barker 2020). In the narrow frequency interval where the frequency spectrum displays a $1 / \omega^{4}$ power law, we find that the eddy viscosity is reduced by the same amount but has positive values. For larger frequencies within the dissipation range, the effective viscosity changes sign and then follows a generic quadratic reduction once $\left|v_{E}\right| \lesssim v$.

For very high frequencies, our results indicate that $\left|v_{E}\right| \propto$ $\left(\left|\omega_{t}\right| / \omega_{c v}\right)^{-2}$ even for these negative values, consistently with asymptotic theory (Ogilvie \& Lesur 2012; Duguid et al. 2020). Moreover, since the change of sign of $v_{E}$ seems to occur when $\left|v_{E}\right| / v \lesssim 1$ in dimensional units (corresponding with frequencies $\left|\omega_{t}\right|$ firmly within the dissipation range), this probably indicates that the observed negative values are not astrophysically relevant but result from (necessarily) adopting simulation parameters that are far removed from their astrophysical values (see below).

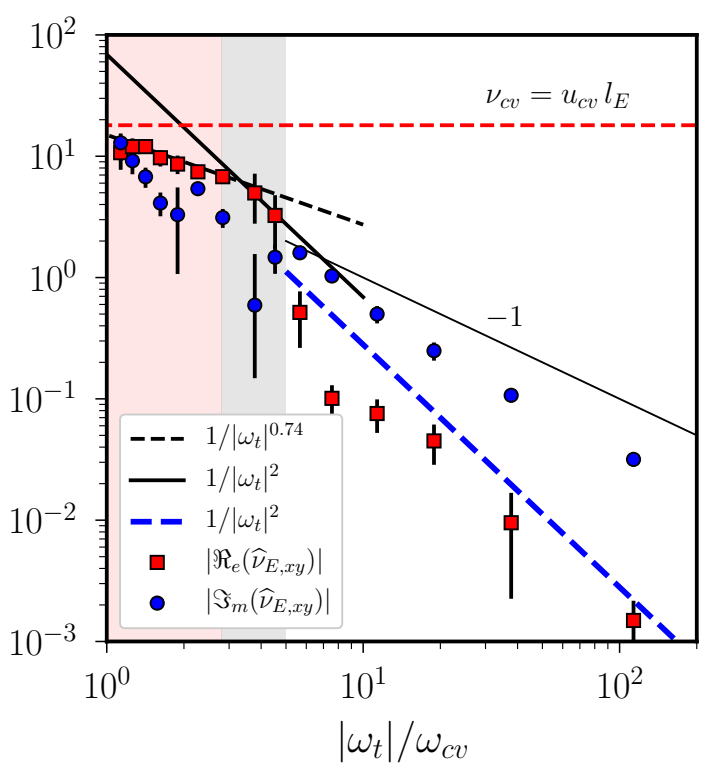

(a) $R a=10^{6}$

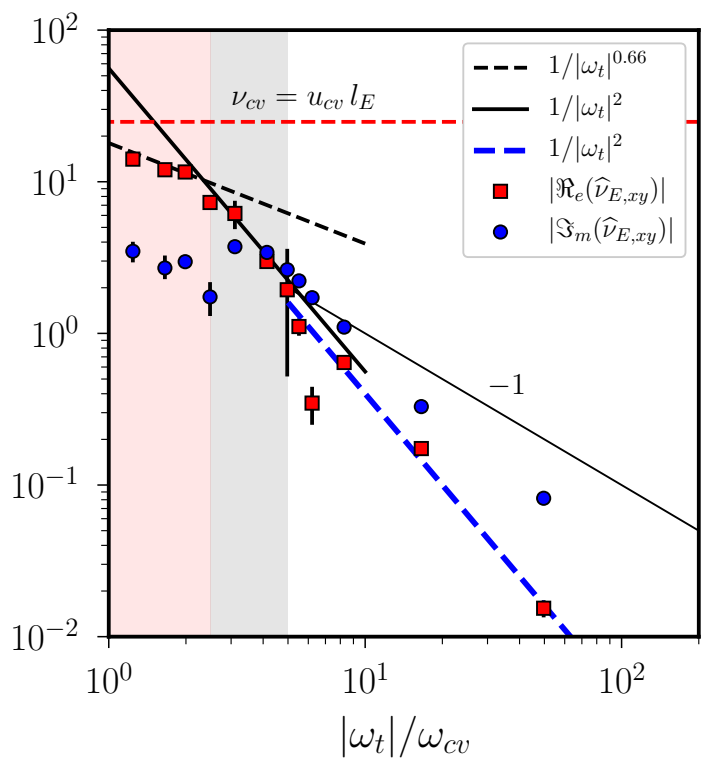

(b) $R a=4 \times 10^{6}$

Figure 9. $\left|\mathfrak{R}_{e}\left(\widehat{v}_{E, x y}\right)\right|$ (red squares) and $\left|\mathfrak{I}_{m}\left(\widehat{v}_{E, x y}\right)\right|$ (blue circles) of the contribution to the effective viscosity defined by equation (9a), as a function of $\left|\omega_{t}\right| / \omega_{c v}$ in DNS with $P r=1$ and $\beta=5 \times 10^{-2}$. Background colors refer to Fig. 5 .

\subsection{Complementary analysis}

We can alternatively compute the effective viscosity associated with each component of the volume-averaged Reynolds stress by relating the stress to the time history of the rate of strain (thus accounting for the oscillatory nature of the tidal flow, see e.g. Ogilvie \& Lesur 2012). In the Fourier domain, this gives

$$
\begin{aligned}
\mathcal{F}\left\{\left\langle u_{x} u_{y}\right\rangle_{V}\right\} & =\widehat{v}_{E, x y} \omega_{t} \beta \mathcal{F}\left\{\cos \left(\omega_{t} t\right)\right\}, \\
\mathcal{F}\left\{\left\langle u_{x}^{2}\right\rangle_{V}\right\} & =\widehat{v}_{E, x x} \omega_{t} \beta \mathcal{F}\left\{\sin \left(\omega_{t} t\right)\right\},
\end{aligned}
$$


and similarly for $\mathcal{F}\left\{\left\langle u_{y}^{2}\right\rangle_{V}\right\}$, where $\left[\widehat{v}_{E, x y}, \widehat{v}_{E, x x}\right]$ are complexvalued quantities.

In the regime of high-frequency tidal forcing $\left(\left|\omega_{t}\right| \gg \omega_{c v}\right)$, Ogilvie \& Lesur (2012) and Duguid et al. (2020) used asymptotic theory to demonstrate the visco-elastic nature of the tidal response (using a simple oscillatory shear) for quantity (9a). In the latter expression, the real part $\mathfrak{R}_{e}\left(\widehat{v}_{E, x y}\right)$ represents a turbulent viscosity (which is in phase with the tidal shear and out of phase with the tidal displacement) associated with this component of the flow, which provides a contribution to the total $v_{E}$. Asymptotic theory indicates that for high frequencies, this quantity should scale as $\left|\omega_{t}\right|^{-2}$ (with possibly negative values). On the other hand, the imaginary part $\mathfrak{J}_{m}\left(\widehat{v}_{E, x y}\right)$ is related to an effective elasticity (which is out of phase with the tidal shear and in phase with the tidal displacement) and should obey a linear reduction $\left|\omega_{t}\right|^{-1}$ in that regime (indicating an effective elastic modulus that is independent of frequency). We show in Fig. 9 direct computations of $\widehat{v}_{E, x y}$ from equation (9a) for two different values of $R a$, which confirm the universal nature of the visco-elastic response of $\left\langle u_{x} u_{y}\right\rangle_{V}$ at high tidal frequencies (here $\left|\omega_{t}\right| / \omega_{c v} \geq O(10)$ ). We broadly obtain a linear reduction $\left|\mathfrak{J}_{m}\left(\widehat{v}_{E, x y}\right)\right| \propto\left|\omega_{t}\right|^{-1}$ in the high-frequency regime, and we also recover the expected scaling in $\left|\omega_{t}\right|^{-2}$ for the turbulent viscosity $\left|\mathfrak{R}_{e}\left(\widehat{v}_{E, x y}\right)\right|$ in this regime. The latter is always smaller than $\left|\mathfrak{J}_{m}\left(\widehat{v}_{E, x y}\right)\right|$, indicating a primarily elastic response to high frequency shear, with a weaker viscous component.

However, this asymptotic theory does not apply for the lower forcing frequencies $\left|\omega_{t}\right| / \omega_{c v} \leq 10$ that we consider here. Indeed, for these lower frequencies, $\left|\mathfrak{R}_{e}\left(\widehat{v}_{E, x y}\right)\right|$ and $\left|\mathfrak{J}_{m}\left(\widehat{v}_{E, x y}\right)\right|$ have comparable magnitudes, and the viscous component can even dominate. Hence, the predictions of the asymptotic theory cannot be strictly invoked to support the quadratic reduction for lower frequencies than those contained in the dissipation range of the convection in our simulations. Instead, we find that the $v_{E}$ behaves similarly to the frequency spectrum of $\left\langle u_{x} u_{y}\right\rangle_{V}$ (e.g. Fig. 6), indicating that this is a key quantity governing the frequency-reduction of the eddy viscosity in our simulations.

We also illustrate in Fig. 10 the contribution to $v_{E}$ from $\mathfrak{J}_{m}\left(\widehat{v}_{E, x x}\right)$ computed from (9b). Similar results are obtained for the $\left\langle u_{y}^{2}\right\rangle_{V}$ component (since $u_{x}$ and $u_{y}$ play symmetrical roles, not shown). The amplitude of the effective viscosity contribution from this component is in broad quantitative agreement with Figs 6 and 9, which cross-validates our computations for the turbulent viscosity. This also agrees with Penev et al. (2009), who showed that the effects of convective turbulence on a large-scale oscillatory shear flow is fairly well represented by an effective viscosity coefficient.

\subsection{Inclusion of weak rotation}

We now introduce global rotation to assess the robustness of the observed frequency-reduction laws for slowly rotating stars or planets. One measure for the degree of rotational constraint in convection-driven flows is given by the convective Rossby number $R o_{c}=E \sqrt{R a / P r}$. Weakly rotating convection is believed to approach non-rotating convection (e.g. Gastine et al. 2016; Long et al. 2020), and so quantitatively similar results are expected for the turbulent viscosity when $R o_{c} \gg 1$ (as considered below). We show in Fig. 11 the DNS with $R o_{c}=10^{2}\left(E=10^{-1}\right)$ and $R o_{c}=10^{1}$ $\left(E=10^{-2}\right)$. By comparison with Fig. 6a, we observe values of $v_{E}$ that are close to the ones obtained in the non-rotating DNS.

Our results indicate here that weak global rotation does not significantly modify the frequency-reduction laws of $v_{E}$ found in

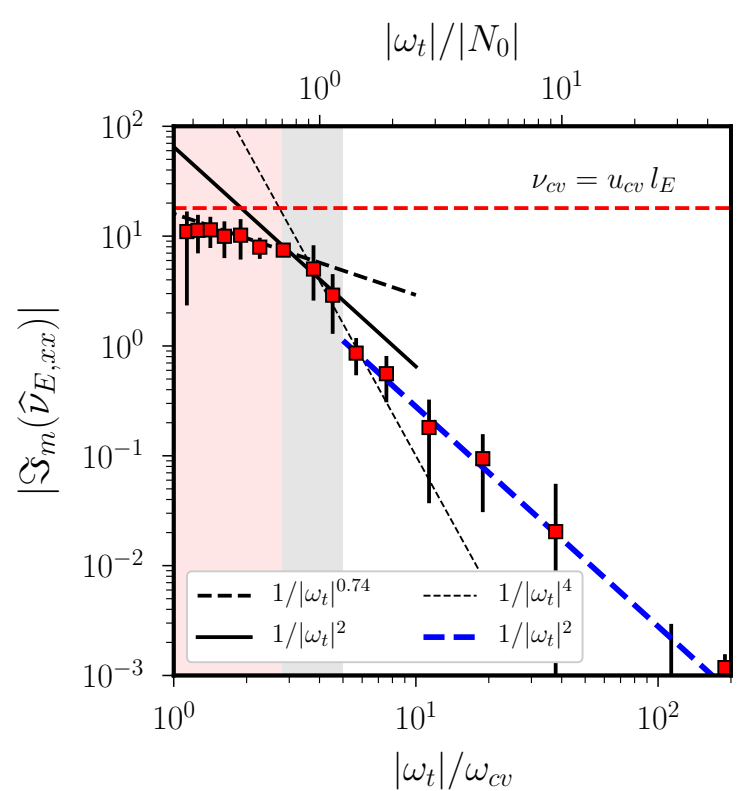

Figure 10. Effective viscosity contribution $\left|\mathfrak{J}_{m}\left(\widehat{v}_{E, x x}\right)\right|$ computed from expression (9b), as a function of $\left|\omega_{t}\right| / \omega_{c v}$ in DNS with $\operatorname{Ra}=10^{6}, \operatorname{Pr}=1$ and $\beta=5 \times 10^{-2}$. Background colors refer to Fig. 5 .

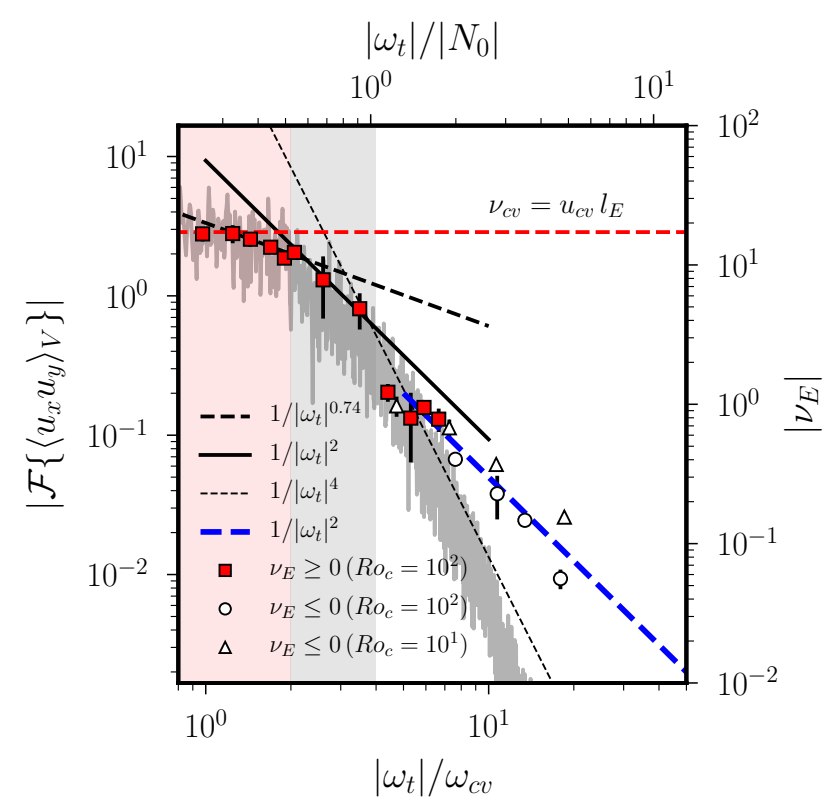

Figure 11. Direct measurements of the effective viscosity $v_{E}$ in weakly rotating DNS with $R a=10^{6}, \operatorname{Pr}=1$ for tidal amplitude $\beta=5 \times 10^{-2}$, as a function of $\left|\omega_{t}\right| / \omega_{c v}$ (or $\left|\omega_{t}\right| /\left|N_{0}\right|$ ). Red squares: $\nu_{E}>0$ in DNS with $R o_{c}=10^{2}\left(E=10^{-1}\right)$ and $\beta=5 \times 10^{-2}$. Empty circles: $v_{E}<0$ in DNS with $R o_{c}=10^{2}\left(E=10^{-1}\right)$ and $\beta=5 \times 10^{-2}$. Empty triangles: $v_{E}<0$ for DNS with $R o_{c}=10^{1}\left(E=10^{-2}\right)$ and $\beta=10^{-2}$. The horizontal dashed lines indicate the MLT expectation $v_{c v} \sim u_{c v} l_{E}$ for $R o_{c}=10^{2}$ $\left(E=10^{-1}\right)$, and the gray curve shows the frequency spectrum of $\left\langle u_{x} u_{y}\right\rangle_{V}$ for unperturbed DNS with $R o_{c}=10^{2}\left(E=10^{-1}\right)$ as a function of $\omega / \omega_{c v}$ (same horizontal axis as $\left|\omega_{t}\right| / \omega_{c v}$ ). Background colors refer to Fig. 5 . 
non-rotating spherical convection. Yet, rapid rotation is known to strongly affect spherical convection (e.g. Guervilly et al. 2019), and is therefore believed to strongly modify the effective viscosity when $R o_{c} \ll 1$ (Mathis et al. 2016). Another complication with incorporating rapid rotation in our model is that the tidal (elliptical) instability can be triggered for large enough $\beta$ when $-1 \leq \Omega_{\text {orb }} / \Omega_{s}=E / E_{\text {orb }} \leq 3$ (Barker et al. 2016; Vidal \& Cébron 2017). Further work is required to explore this regime, which might be relevant for giant planets or young rapidly rotating stars.

\section{DISCUSSION}

\subsection{Non-Kolmogorov turbulent spectrum}

Our DNS have shown that the frequency-reduction of the eddy viscosity is directly correlated with the frequency spectrum of the convection (which is largely unaltered by the tidal flow). Outside the dissipation range of the convection, we have recovered the quadratic reduction for frequencies in the Kolmogorov cascade (Goldreich \& Nicholson 1977), but for lower frequencies where the frequency spectrum is less steep than the Kolmogorov spectrum, we have found a new frequency reduction that is surprisingly smaller than the linear suppression proposed by Zahn (1966). One could look at the scales that dominate the effective viscosity to get further physical insight into this problem. Zahn (1966) indeed assumed that the dissipation is dominated by the largest eddies, whereas Goldreich \& Nicholson (1977) assumed that the 'resonant eddies' dominate the dissipation.

To this end, we illustrate in Fig. 12 the radial dependence of the turbulent viscosity for the illustrative DNS with $R a=10^{6}$ and $\beta=5 \times 10^{-2}$ for different tidal frequencies. We show the power spectrum (normalized by its maximum value) of the $l=m=0$ component (i.e. the surface-average per shell) of quantity (8a) as a function of the radius $r$. Within the anomalous range $\left(\left|\omega_{t}\right| / \omega_{c v} \gtrsim 1\right.$ in Figs $12 \mathrm{a}$ and $\mathrm{b}$ ), the eddy viscosity is dominated by turbulent eddies deep in the interior. We also find a significant contribution of the interior eddies in Fig. 12d, for DNS with much higher frequencies $\left|\omega_{t}\right| / \omega_{c v} \geq 10$ (i.e. characterized by the quadratic suppression with negative values), but smaller-scale turbulent interactions are also triggered nearer the surface (except in the outer thin thermal boundary layer). These radial profiles do not allow us to disentangle easily the length scales that are responsible for the various scaling laws for $v_{E}$. However, they do show a tendency for larger radii to contribute more at high frequencies. This trend might be expected if the 'resonant eddies' at each radius (with frequencies comparable with $\left.\left|\omega_{t}\right|\right)$ are important, since the convective heat flux increases with radius such that the local convective eddies have larger frequencies nearer the surface. However, our simulations do not provide convincing support for this hypothesis (see also in Duguid et al. 2020).

In light of our findings, we have revisited the numerical results of Penev et al. (2009) from an independent viewpoint. Indeed, they argued that the observed linear scaling for the effective viscosity in their DNS was due to the shallower than Kolmogorov frequency spectrum of the convection. Hence, one might wonder whether their DNS were subject to $1 / \omega^{\alpha}$ dynamics (as found in our DNS). We reproduce in Fig. 13 the frequency power spectrum of the convective flows in their DNS. The spectra are less steep than the expected Kolmogorov spectrum $1 / \omega^{2}$, in broad agreement with the power law $1 / \omega^{\delta}$ with $^{2} \delta \approx 1.2$. The latter value is incompatible with our results, since we have always found $1 / \omega^{\alpha}$ power laws with $\alpha<1$ within the anomalous range. Instead, the reduction factor for $v_{E}$ obtained by Penev et al. (2009) could result from eddies in a turbulent cascade (as in Goldreich \& Nicholson 1977), but only if the theoretical scaling for $v_{E}$ is modified to account for spatial spectra with non-standard power exponents $(\neq-5 / 3)$ in the turbulent cascade.

Indeed, a simple predictive theory can be developed for incompressible flows, which relates the power exponent $\delta$ of the frequency spectrum to the power exponent $\Lambda$ of the spatial spectrum of the turbulent kinetic energy, such that (Goldman \& Mazeh 1991)

$\Lambda=\frac{3 \delta-1}{1+\delta}, \quad \delta=\frac{1+\Lambda}{3-\Lambda}$

Standard Kolmogorov turbulence with $\Lambda=5 / 3$ gives $\delta=2$ (Landau \& Lifshitz 1987), as considered by Goldreich \& Nicholson (1977). We can then deduce from (10) that the frequency-reduction of the eddy viscosity is $v_{E} \propto 1 /\left|\omega_{t}\right|^{\delta}$ (see the derivation Appendix A in Goldman \& Mazeh 1991). Note that the $1 / \omega^{\alpha}$ spectra observed in our DNS with $\alpha<1$ cannot be explained by the latter theory, since the spatial exponent $\Lambda$ predicted by (10a) that is required to match $\delta<1$ does not agree with the observed spatial spectra in Fig. 2. A non-Kolmogorov "cascade" with $\Lambda \neq 5 / 3$ could be produced by scale-dependent buoyant driving or non-negligible viscous damping, and it might also result from anisotropic or inhomogeneous turbulence.

We show in Fig. 14 the time-averaged spatial power spectra of the velocity components reported in Penev et al. (2009). To be more consistent with the incompressible theory, we have only shown the power spectra of the horizontal velocity components as a function of the horizontal wave numbers $k_{x}$ and $k_{y}$ (since their anelastic results could differ more importantly from this simple incompressible theory in the vertical direction, as a result of their adopted density stratification). The spatial spectra, which are clearly flatter than the Kolmogorov spectrum (i.e. with $\Lambda \leq 5 / 3$ ), are in good agreement with the power law $k_{i}^{-\Lambda}$ with the exponent $\Lambda=1.18$ given by expression (10a) assuming $\delta=1.2$ (see Fig. 13).

Finally, we reproduce in Fig. 15 the horizontal effective eddy viscosity coefficient, computed from the DNS of Penev et al. (2009), as a function of $\left(\left|\omega_{t}\right| / \omega_{c v}\right)^{-1}$ using Penev's representation. Even if the measurements are subject to relatively large uncertainties, the frequency-reduction of the eddy viscosity in the fast tide range (here $\left.\left(\left|\omega_{t}\right| / \omega_{c v}\right)^{-1} \leq 1\right)$ is in good agreement with our prediction using equation (10) assuming $\delta=1.2$. Moreover, our theory is also more consistent with the fact that the eddies with convective time-scales close to the tidal forcing period were responsible for most of the dissipation in the compressible DNS, as reported by Penev et al. (2009) (contrary to Zahn's assumption). Therefore, the fact that frequency-reduction law reported in Penev et al. (2009) appears broadly consistent with a linear suppression cannot be taken to conclusively support Zahn's prescription.

To summarize, very different frequency spectra can be generated by turbulent convection, leading to different prescriptions for the frequency-suppression law of the eddy viscosity. They can manifest in the form of anomalous $1 / \omega^{\alpha}$ power laws for low to intermediate frequencies, such that the frequency-reduction law of the eddy viscosity is expected to be directly correlated with the anomalous frequency spectrum (as reported here). Additionally,

2 The exponent $\delta$ given in fig. 3 has a typo in Penev et al. (2009), which has been corrected in Penev et al. (2011) 


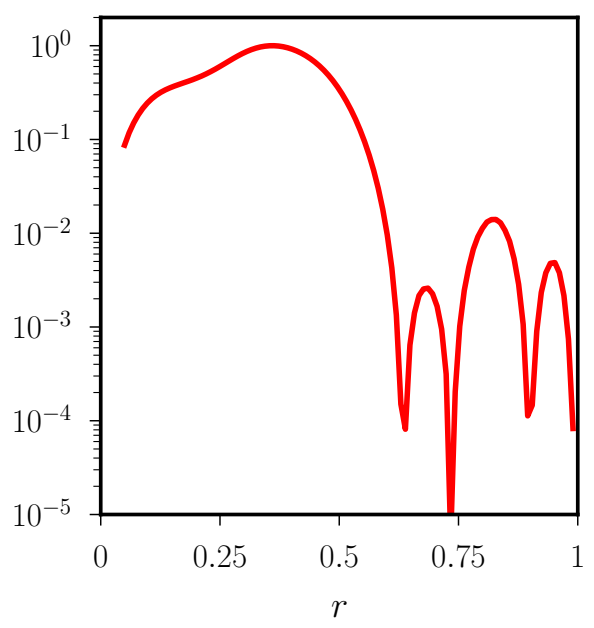

(a) $\left|\omega_{t}\right| / \omega_{c v}=1.13$

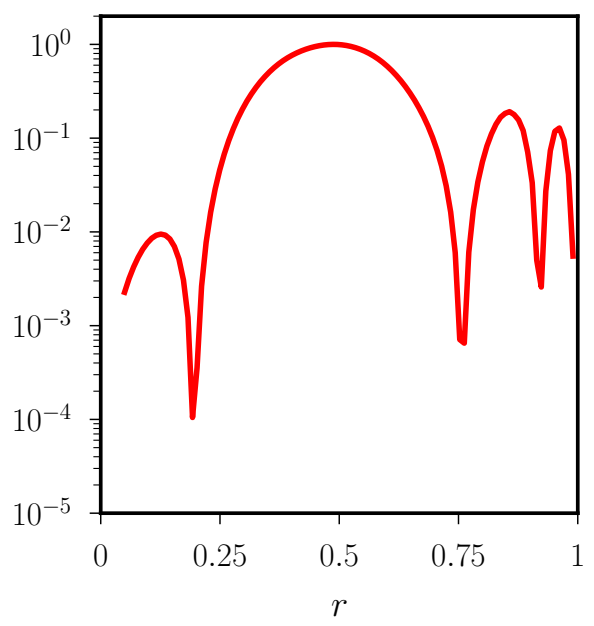

(c) $\left|\omega_{t}\right| / \omega_{c v}=5.67$

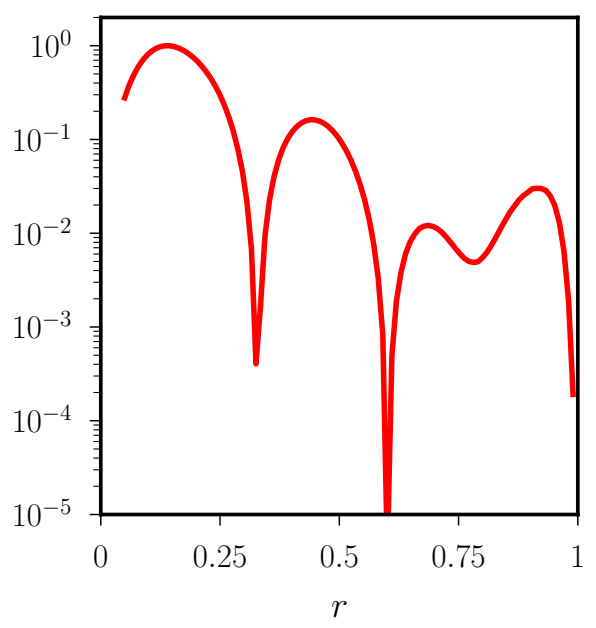

(b) $\left|\omega_{t}\right| / \omega_{c v}=1.89$

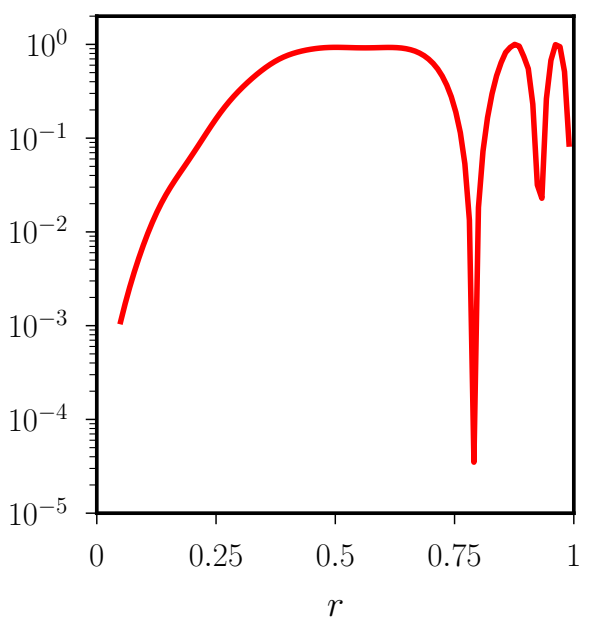

(d) $\left|\omega_{t}\right| / \omega_{c v}=11.3$

Figure 12. Normalized power spectrum of the time-averaged $l=m=0$ component (i.e. average on a spherical shell) of equation (8a) as a function of radius $r$ in DNS with $\operatorname{Ra}=10^{6}, \mathrm{Pr}=1$ and $\beta=5 \times 10^{-2}$. These indicate the radii that provide the dominant contribution to $v_{E}$.

the convection can also exhibit a turbulent cascade that is less steep than the Kolmogorov spectrum (e.g. Penev et al. 2009), such that the quadratic reduction factor of the eddy viscosity initially proposed by Goldreich \& Nicholson (1977) ought to be modified accordingly.

\subsection{Astrophysical implications}

Our findings indicate that the frequency dependence of the eddy viscosity is surprisingly much more complex than initially proposed by Zahn (1966) and Goldreich \& Nicholson (1977). We can qualitatively extrapolate our findings to weakly rotating stellar interiors as illustrated in Fig. 16. For very low frequency forcing, standard expectations from MLT (e.g. Spiegel 1971) predict the eddy viscosity to scale as $v_{E} \sim u_{c v} l_{E} \propto(R a / P r)^{1 / 2}$ in dimensionless units, independently of the tidal frequency when $\left|\omega_{t}\right| / \omega_{c v} \ll 1$. The latter scaling is consistent with constant tidal lag-time models (e.g. Alexander 1973; Hut 1981; Eggleton et al. 1998), which are commonly applied in astrophysics. However, since this model is only valid for very low tidal frequencies $\left(\left|\omega_{t}\right| \ll \omega_{c v}\right)$, the con- stant time-lag model should not be used for the majority of tidal applications, particularly those in which $\left|\omega_{t}\right| \gtrsim \omega_{c v}$.

In the presence of fast tides $\left|\omega_{t}\right| / \omega_{c v} \geq 1$, the effective viscosity ought to be reduced. A $1 /\left|\omega_{t}\right|^{\alpha}$ power-law reduction is first expected, with shallow exponents $\alpha<1$. Secondly, for frequencies in a turbulent cascade that is characterized by a power-law spatial spectrum with an arbitrary exponent $\Lambda$, the effective viscosity should be reduced as $v_{E} \propto 1 /\left|\omega_{t}\right|^{(1+\Lambda) /(3-\Lambda)}$ (Goldman \& Mazeh 1991). This gives a quadratic reduction for standard Kolmogorov turbulence (as proposed by Goldreich \& Nicholson 1977). This quadratic reduction is probably the relevant one in stars and planets (e.g. Goldreich \& Keeley 1977), but further work is required to assess this hypothesis with more realistic compressible (or anelastic) models. Finally, for much higher frequencies, the eddy viscosity may exhibit a quartic reduction $v_{E} \propto 1 /\left|\omega_{t}\right|^{4}$ in a narrow transition range towards the dissipation scales of the turbulence, and then a quadratic suppression $\left|v_{E}\right| \propto 1 /\left|\omega_{t}\right|^{2}$ with possibly negative values for tidal frequencies further into the dissipation range when $\left|v_{E}\right| \lesssim v$ (see also in Ogilvie \& Lesur 2012; Duguid et al. 2020).

Based on our results, robust quantitative extrapolation is cur- 


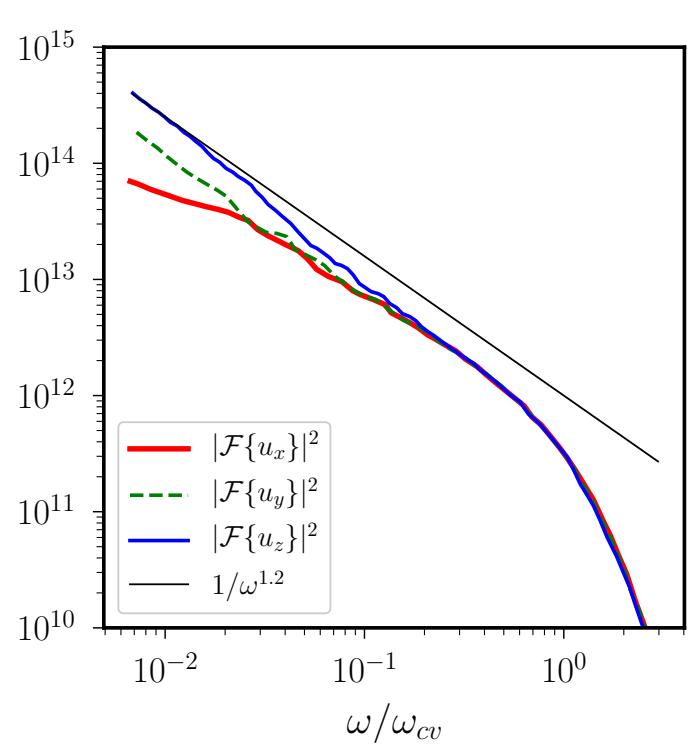

Figure 13. Frequency spectra of the three velocity components $\left[u_{x}, u_{y}, u_{z}\right]$ in anelastic DNS, obtained from fig. 3 in Penev et al. (2009).

rently challenging beyond the aforementioned qualitative picture. The latter two frequency regimes may be not relevant in astrophysics, because they would require very large values of $\left|\omega_{t}\right| / \omega_{c v}$. MLT indeed predicts $\omega_{c v} \propto(\operatorname{Ra} / P r)^{1 / 2}$ in the fully turbulent regime (in broad agreement with our DNS, as shown in the inset panel in Fig. 4). For solar-like stars, typical values for the Rayleigh and Prandtl numbers are indeed $R a=10^{19}-10^{24}$ and $\operatorname{Pr}=10^{-6}-10^{-4}$ (Hanasoge \& Sreenivasan 2014), such that the turbulent cascade should extend to much higher frequencies, and the lower bound of the dissipation range should be shifted to $\left|\omega_{t}\right| / \omega_{c v} \gg O(10)$, compared with our simulations. Values $v_{E} \gg v$ are thus expected in most stellar interiors. Negative values $v_{E} \leq 0$ may be theoretically possible in stellar interiors, but very large values of $\left|\omega_{t}\right|$ would probably be required, which are likely to be unrealistic for large-scale tidal flows. The turbulent convective damping of the acoustic modes (Goldreich \& Keeley 1977) also provides an indirect viewpoint that may suggest that the observed negative values are not physically relevant. Indeed, if the observed correlation between the frequency spectrum of the convection and the frequency-reduction law of the eddy viscosity is generic, then the turbulent cascade should extend until very large frequencies ${ }^{3}$ because a quadratic reduction of positive eddy viscosities is probably required to explain the damping of these modes (e.g. Goldreich et al. 1994; Samadi et al. 2001).

The power spectrum observed within the anomalous range may naively appear as a transition between the zero-frequency and the Kolmogorov-like scalings (as in Goldman 2008). However, this is more probably an occurrence of $1 / \omega^{\alpha}$ turbulent noise (Niemann et al. 2013), which is a robust feature of various turbulent flows (e.g. Herault et al. 2015a; Pereira et al. 2019). This power spectrum may thus exist in turbulent stellar (or planetary) interiors, resulting from the long-term properties of the turbulent flows (according to prior statistical theories, e.g. Herault et al. 2015b). We have

\footnotetext{
3 The acoustic modes have much larger frequencies than those of tidal
} forcing.

MNRAS 000, 1-14 (2020)
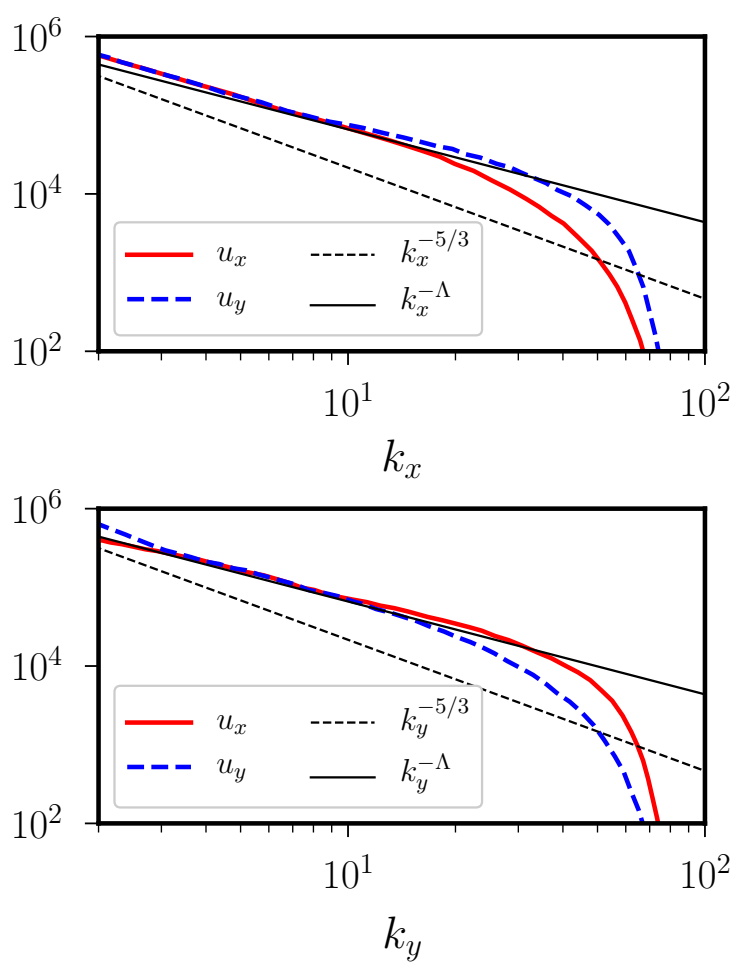

Figure 14. Time-averaged horizontal power spectrum of the velocity components $\left[u_{x}, u_{y}\right]$ in anelastic DNS (obtained from the top panels of fig. 3 in Penev et al. 2009), as a function of the horizontal wave numbers $\left[k_{x}, k_{y}\right]$ in plane-layer geometry.

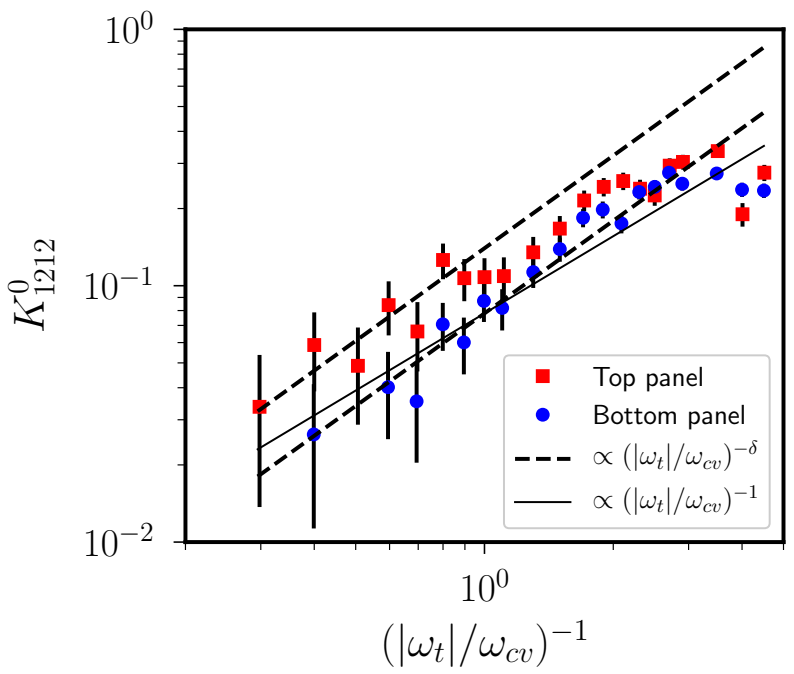

Figure 15. Comparison between theory (10), linear reduction, and direct measurements of the eddy viscosity (denoted here $K_{1212}^{0}$ ) in anelastic DNS (extracted from the weak forcing case of fig. 12 in Penev et al. 2009). The two data sets (red squares and blue circles) have been computed using two different methods (see details in Penev et al. 2009). Two power laws $K_{1212}^{0} \propto\left(\left|\omega_{t}\right| / \omega_{c v}\right)^{-\delta}$ with $\delta=1.2$ have been drawn (one for each data set in the range $\left.\left(\left|\omega_{t}\right| / \omega_{c v}\right)^{-1} \leq 1\right)$. 


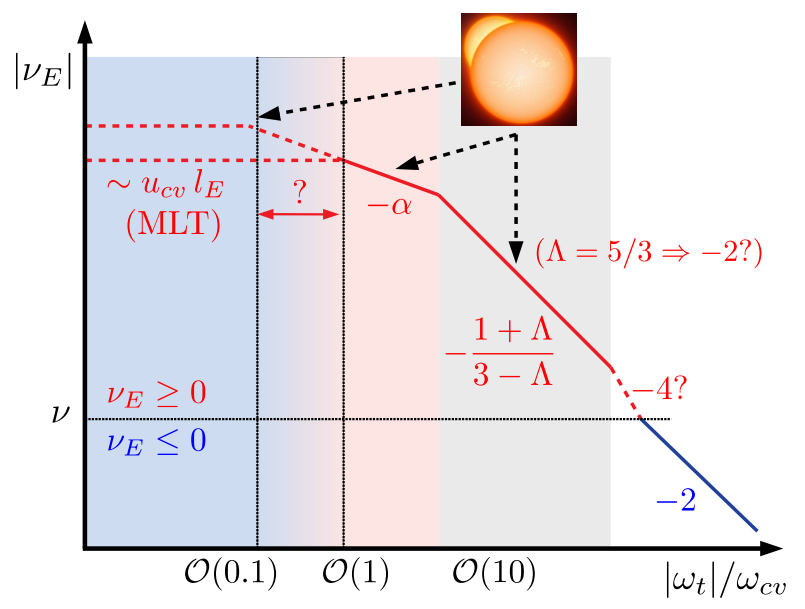

Figure 16. Expected behavior of $v_{E}$ as a function of $\left|\omega_{t}\right| / \omega_{c v}$ in turbulent stellar interiors. Laminar viscosity $v$, turbulent viscosity $v_{E} \sim u_{c v} l_{E}$ (MLT) in the low-frequency regime. $\Lambda$ is the power exponent of the spatial spectrum of the kinetic energy. Background colors refer to Fig. 5.

unfortunately found here power exponents $\alpha<1$ that vary with $R a$ and $P r$ in full spheres (see the slopes in Figs 6a and 7, both obtained at $R a=10^{6}$ ), contrary to preliminary findings in planelayer geometries (with $\alpha \simeq 0.5$, which will be presented elsewhere). This indicates an important model-dependence to the anomalous range, and so we cannot currently extrapolate the numerical values of $\alpha$ for very turbulent interiors.

Our DNS also suggest that $v_{E}$ could be reduced (over its low frequency asymptotic value) for smaller frequencies $\left|\omega_{t}\right| / \omega_{c v} \lesssim 1$ as indicated in Fig. 16, because the anomalous range may extend until $\left|\omega_{t}\right| / \omega_{c v} \simeq O(0.1)$ or perhaps below (as observed in the various frequency spectra). Yet, since computations of the lowfrequency spectrum of turbulent flows are very challenging, we have been unable to directly measure the eddy viscosity within the low-frequency regime, and we do not have very reliable estimates of the transition values between the two regimes for very turbulent stellar interiors.

To illustrate one of the uncertainties in applying our results, we briefly explore how the theoretical time-scales for binary spin synchronization are affected by changes in the slope $\alpha$ of the anomalous regime. To do so, we consider a continuous piece-wise power-law profile for $v_{E}$ based on our simulations (as illustrated in Fig. 16). We adopt $v_{E}=u_{c v} l_{E}$ for $\omega_{t} / \omega_{c v} \leq 0.3$, then $v_{E} \propto 1 /\left|\omega_{t}\right|^{\alpha}$ for $0.3<\left|\omega_{t}\right| / \omega_{c v}<3$, and finally $v_{E} \propto 1 /\left|\omega_{t}\right|^{2}$ when $\left|\omega_{t}\right| / \omega_{c v} \geq 3$ (discarding the possible negative values of $v_{E}$ ). We consider the values $0.5 \leq \alpha \leq 1$ that span our simulations. We use main-sequence stellar models computed with MESA (see Appendix A for further details), where $u_{c v}$ and $l_{E}$ are here the convective velocity and mixing length that vary with stellar radius, and $\omega_{c v}=u_{c v} / l_{E}$. We calculate the correct equilibrium tide in convective regions (Terquem et al. 1998; Ogilvie 2014), which differs from the commonly-adopted but strictly incorrect equilibrium tide of Zahn (1989), and then compute the dissipation integral. We thus obtain a tidal quality factor $Q_{\mathrm{eq}}^{\prime}$, from which the time-scale for tidal synchronization of the stellar spin of the primary star interacting with a companion of mass $M_{2}$ is (after correcting a typographical error in formula (7) of Vidal \& Barker 2020)

$\tau_{\Omega}=\frac{2 Q_{\mathrm{eq}}^{\prime}}{9 \pi r_{g}^{2}}\left(\frac{M+M_{2}}{M_{2}}\right)^{2} \frac{P_{o r b}^{4}}{P_{d y n}^{2} P_{s}}$,

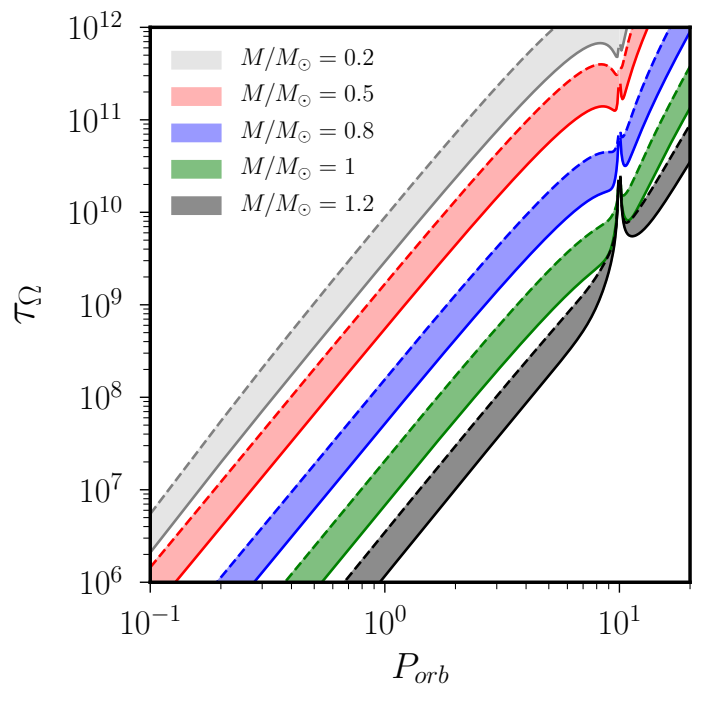

Figure 17. Synchronization time-scale $\tau_{\Omega}$ (in years), as a function of $P_{o r b}$ (in days), due to convective damping of the equilibrium tide (e.g. Terquem et al. 1998; Ogilvie 2014), for a star of mass $M$ with the initial spin period $P_{S}=10 \mathrm{~d}$. The companion has a fixed mass $M_{2}=M_{\odot}$. Viscosity prescription based on Fig. 16 with $0.5 \leq \alpha \leq 1$ within the anomalous range $0.3<\left|\omega_{t}\right| / \omega_{c v}<3$, and a quadratic reduction when $\left|\omega_{t}\right| / \omega_{c v} \geq 3$ (i.e. with $\Lambda=5 / 3$ ). Solid lines (respectively dashed lines) have been computed with $\alpha=0.5$ (respectively $\alpha=1$ ).

where $r_{g}^{2}$ is the dimensionless squared radius of gyration, $P_{d y n}=$ $2 \pi /\left(G M / R^{3}\right)^{1 / 2}$ is the dynamical time-scale, $P_{\text {or } b}=2 \pi / \Omega_{\text {orb }}$ is the orbital period, and $P_{S}=2 \pi / \Omega_{S}$ is the (initial) spin period. We show in Fig. 17 the results for $\tau_{\Omega}$ as a function of $P_{\text {orb }}$ with $M_{2}=M_{\odot}$ (where $M_{\odot}$ is the solar mass) and $P_{S}=10 \mathrm{~d}$ in each case, for a range of main-sequence stellar models with masses $M / M_{\odot} \in[0.2,0.5,0.8,1.0,1.2]$ that correspond to the stellar ages $[2.9,3.3,2.6,4.7,2.9]$ Gyr. This shows that for an anomalous regime spanning a decade in frequency, uncertainties in $\alpha$ only affect $\tau_{\Omega}$ by a factor of two or three (except near spin-orbit synchronization at $P_{\text {orb }}=P_{s}=10$ ). On the other hand, if the anomalous range is much wider, uncertainties in $\alpha$ could have more important effects on $\tau_{\Omega}$ (not shown).

Finally, the power spectrum of the turbulent cascade is also uncertain. Kolmogorov spectra have been robustly reported for Boussinesq convection (Kumar \& Verma 2018), but compressible convection (e.g. Penev et al. 2011; Horst et al. 2020) may display different non-Kolmogorov spectra (depending on the convection setup). Further work is required to characterize the frequency spectrum of more realistic stellar convection so that we can robustly apply our results to astrophysical tidal evolution.

\section{CONCLUDING REMARKS}

In this paper, we have revisited numerically the long-standing controversy regarding the interaction between equilibrium tidal flows and turbulent convection. We have conducted DNS of thermal convection within an idealized global model of a fully-convective fluid body, which is a simple analogue of a low-mass star or core-less giant planet, to measure the turbulent viscosity $v_{E}$ acting on the large-scale equilibrium tidal flow.

Our results have highlighted that quantifying the efficiency of 
tidal dissipation resulting from this mechanism is more complex than was previously believed. Indeed, we have found that neither the often-disputed linear (Zahn 1966) or quadratic (Goldreich \& Nicholson 1977) scaling laws for the turbulent viscosity are generally valid for tidal frequencies $\left|\omega_{t}\right|$ that exceed the dominant convective turnover frequency $\omega_{c v}$. Instead, we have demonstrated that the frequency-reduction law of the eddy viscosity is correlated with the frequency spectrum of the unperturbed convection, and we have obtained here various scaling laws in our DNS.

The eddy viscosity is first reduced as $v_{E} \propto 1 /\left|\omega_{t}\right|^{\alpha}$ for tidal frequencies below those in the turbulent cascade, with shallow exponents $\alpha<1$. Then, for frequencies in a turbulent Kolmogorov-like cascade with the spatial power exponent $\Lambda$, we have consistently combined our numerical findings with Penev's previous results to show that $v_{E} \propto 1 /\left|\omega_{t}\right|^{(1+\Lambda) /(3-\Lambda)}$. For the standard Kolmogorov cascade with $\Lambda=5 / 3$, which is probably the relevant one over a broad range of scales in stars or planets (as supported by observations of solar acoustic modes, e.g. Goldreich \& Keeley 1977; Samadi et al. 2001), this leads to $v_{E} \propto 1 /\left|\omega_{t}\right|^{2}$ (Goldreich \& Nicholson 1977). However, further work is required to explore the robustness of this scaling in more realistic (compressible or anelastic) models. Our results finally support the universality of the quadratic reduction law $\left|v_{E}\right| \propto\left|\omega_{t}\right|^{-2}$ for very high frequencies in the dissipation range of the convection, which is consistent with asymptotic predictions when $\left|\omega_{t}\right| / \omega_{c v} \gg 1$ (Ogilvie \& Lesur 2012; Duguid et al. 2020).

Our findings have important consequences for interpreting astrophysical observations such as those that constrain tidal synchronization and circularization of main-sequence binaries (e.g. Meibom \& Mathieu 2005; Meibom et al. 2006; Van Eylen et al. 2016; Lurie et al. 2017; Triaud et al. 2017) and the circularization of evolved stars (Verbunt \& Phinney 1995; Beck et al. 2018; PriceWhelan \& Goodman 2018). Indeed, it appears that a fundamental knowledge of stellar convection is required before we can be confident in modeling the tidal evolution of astrophysical systems due to this mechanism. Hence, further work is required to understand the properties of more realistic convection models in the presence of oscillatory tidal flows. The transitions between the various regimes observed in our DNS remain for instance poorly constrained, since we have necessarily adopted simulation parameters that are far removed from their astrophysical values, and so should be further explored in more realistic models of stellar convection. The anomalous $1 / \omega^{\alpha}$ spectrum should be also further investigated as a function of $R a / P r$, as well as the slope of power spectrum of the turbulent cascade, which would be very challenging numerically in more turbulent setups. Astrophysical extrapolations also employ crude applications of MLT to the low-frequency regime, which is known not to be accurate in detail (e.g. Goldman 2008) and departures from MLT have been found in DNS of compressible convection (e.g. Anders et al. 2019). Hence, MLT predictions should be carefully compared to more turbulent DNS of convection.

We have considered only circular orbits in this paper, but different tidal components generally coexist (e.g. for eccentric orbits, see in Ivanov \& Papaloizou 2004; Vick \& Lai 2020) and they could be damped at different rates (e.g. Lai 2012). We have also neglected dynamical tides (e.g. Ogilvie \& Lin 2007), although their interaction with convection may be important when inertial waves are excited. Indeed, tidally-excited inertial waves (restored by Coriolis forces) may be the key driver of binary circularization and synchronization in sufficiently rapidly rotating stars (e.g. Ogilvie \& Lin 2007; Goodman \& Lackner 2009; Ivanov et al. 2013; Favier et al. 2014).

Finally, note that our simple physical picture should remain qualitatively valid in weakly rotating interiors (i.e. slow rotators).
However, rapid rotation is known to strongly affect convectiondriven turbulence, as reported in DNS of plane-layer (e.g. Barker et al. 2014; Currie et al. 2020) and spherical convection (e.g. Kaplan et al. 2017; Guervilly et al. 2019), and it has also been proposed that it could modify the effective viscosity (Mathis et al. 2016). Further work is required to explore rapidly rotating convection, which might be relevant for giant planets or young rapidly rotating stars. Since the frequency spectrum of the convection could be strongly impacted by rapid global rotation, the interactions between tidal flows and convection is worth investigating for these applications. Non-linear tidal flows can also be triggered in rapidly rotating interiors for sufficiently large tidal deformations (such as the elliptical (tidal) instability, e.g. Barker et al. 2016; Vidal \& Cébron 2017), which could enhance tidal dissipation for the shortest orbital periods (Barker 2016; Vidal et al. 2018, 2019). Understanding the interplay of these flows with convection also deserves future work.

\section{ACKNOWLEDGEMENTS}

We acknowledge the referee, Adam S. Jermyn, for a prompt report that helped us to significantly improve the clarity of the paper. This work was funded by STFC Grant ST/R00059X/1. This work used the DiRAC@Durham facility managed by the Institute for Computational Cosmology on behalf of the STFC DiRAC HPC Facility. The equipment was funded by BEIS capital funding via STFC capital grants ST/P002293/1, ST/R002371/1 and ST/S002502/1, Durham University and STFC operations grant ST/R000832/1. DiRAC is part of the National e-Infrastructure. Some DNS were also performed using the UKMHD1-UKMHD2-UKMHD3 allocations on the DiRAC Data Intensive service at Leicester, operated by the University of Leicester IT Services, which forms part of the STFC DiRAC High-Performance Computing (HPC) Facility. Postprocessing was performed on ARC4, part of the HPC facilities at the University of Leeds (UK).

\section{DATA AVAILABILITY}

The python scripts and post-processed data underlying the figures are provided as supplementary materials. Data underlying the Figs 13-15 have been extracted from the original figures of Penev et al. (2009) using the online tool https://apps automeris. io/wpd/. The spherical harmonics analysis has been performed using the open-source library shtNs (Schaeffer 2013), available at https://bitbucket.org/nschaeff/shtns/. The DNS underlying this article can be replicated using the open-source code NeK5000, available at https://nek5000.mcs .anl .gov/.

\section{REFERENCES}

Alexander M. E., 1973, Ap\&SS, 23, 459

Anders E. H., Lecoanet D., Brown B. P., 2019, ApJ, 884, 65

Barker A. J., 2016, MNRAS, 459, 939

Barker A. J., Lithwick Y., 2013, MNRAS, 435, 3614

Barker A. J., Dempsey A. M., Lithwick Y., 2014, ApJ, 791, 13

Barker A. J., Braviner H. J., Ogilvie G. I., 2016, MNRAS, 459, 924

Beck P. G., Mathis S., Gallet F., Charbonnel C., Benbakoura M., García R. A .and doÂăNascimento J.-D., 2018, MNRAS, 479, L123

Braviner H. J., 2016, PhD thesis, Univ. Cambridge

Currie L. K., Barker A. J., Lithwick Y., Browning M. K., 2020, MNRAS, 493, 5233

Duguid C. D., Barker A. J., Jones C. A., 2020, MNRAS, 491, 923 
Eggleton P. P., Kiseleva L. G., Hut P., 1998, ApJ, 499, 853

Favier B., Barker A. J., Baruteau C., Ogilvie G. I., 2014, MNRAS, 439, 845

Fischer P. F., Loth F., Lee S. E., Lee S.-W., Smith D. S., Bassiouny H. S., 2007, Comput. Methods Appl. Mech. Eng., 196, 3049

Gastine T., Wicht J., Aubert J., 2016, J. Fluid Mech., 808, 690

Goldman I., 2008, Astron. Nachr., 329, 762

Goldman I., Mazeh T., 1991, ApJ, 376, 260

Goldreich P., Keeley D. A., 1977, ApJ, 211, 934

Goldreich P., Nicholson P. D., 1977, Icarus, 30, 301

Goldreich P., Murray N., Kumar P., 1994, ApJ, 424, 466

Gonczi G., 1982, A\&A, 110, 1

Goodman J., Lackner C., 2009, ApJ, 696, 2054

Goodman J., Oh S. P., 1997, ApJ, 486, 403

Guervilly C., Cardin P., Schaeffer N., 2019, Nature, 570, 368

Hanasoge S. M., Sreenivasan K., 2014, Sol. Phys., 289, 3403

Herault J., Pétrélis F., Fauve S., 2015a, Europhys. Lett., 111, 44002

Herault J., Pétrélis F., Fauve S., 2015b, J. Stat. Phys., 161, 1379

Horst L., Edelmann P. V. F., Andrassy R., Roepke F. K., Bowman D. M., Aerts C., Ratnasingam R. P., 2020, arXiv preprint

Hut P., 1981, A\&A, 99, 126

Ivanov P. B., Papaloizou J. C. B., 2004, MNRAS, 353, 1161

Ivanov P. B., Papaloizou J. C. B., Chernov S. V., 2013, MNRAS, 432, 2339

Kaplan E. J., Schaeffer N., Vidal J., Cardin P., 2017, Phys. Rev. Lett., 119, 094501

Kirk B., et al., 2016, ApJ, 151, 68

Kumar A., Verma M. K., 2018, RSOS, 5, 172152

Lai D., 2012, MNRAS, 423, 486

Lai D., Rasio F. A., Shapiro S. L., 1993, ApJS, 88, 205

Landau L. D., Lifshitz E. M., 1987, Theoretical Physics. Fluid Mechanics, second edn. Pergamon Press, Oxford

Le Bars M., Cébron D., Le Gal P., 2015, Annu. Rev. Fluid. Mech., 47, 163

Liot O., et al., 2016, J. Fluid Mech., 794, 655

Long R. S., Mound J. E., Davies C. J., Tobias S. M., 2020, J. Fluid Mech., 889, A7

Lurie J. C., et al., 2017, ApJ, 154, 250

Mathis S., Auclair-Desrotour P., Guenel M., Gallet F., Le Poncin-Lafitte C., 2016, A\&A, 592, A33

Mazeh T., 2008, EAS, 29, 1

Meibom S., Mathieu R. D., 2005, ApJ, 620, 970

Meibom S., Mathieu R. D., Stassun K. G., 2006, ApJ, 653, 621

Monville R., Vidal J., Cébron D., Schaeffer N., 2019, Geophys. J. Int., 219, S195

Newton E. R., Mondrik N., Irwin J., Winters J. G., Charbonneau D., 2018, ApJ, 156, 217

Nielsen M. B., Gizon L., Schunker H., Karoff C., 2013, A\&A, 557, L10

Niemann M., Kantz H., Barkai E., 2013, Phys. Rev. Lett., 110, 140603

Ogilvie G. I., 2014, ARA\&A, 52, 171

Ogilvie G. I., Lesur G., 2012, MNRAS, 422, 1975

Ogilvie G. I., Lin D. N. C., 2007, ApJ, 661, 1180

Paxton B., Bildsten L., Dotter A., Herwig F., Lesaffre P., Timmes F., 2011, ApJS, 192, 3

Paxton B., et al., 2013, ApJS, 208, 4

Paxton B., et al., 2015, ApJS, 220, 15

Paxton B., et al., 2018, ApJS, 234, 34

Paxton B., et al., 2019, ApJS, 243, 10

Penev K., Sasselov D., Robinson F., Demarque P., 2007, ApJ, 655, 1166

Penev K., Barranco J., Sasselov D., 2009, ApJ, 705, 285

Penev K., Barranco J., Sasselov D., 2011, ApJ, 734, 118

Pereira M., Gissinger C., Fauve S., 2019, Phys. Rev. E, 99, 023106

Price-Whelan A. M., Goodman J., 2018, ApJ, 867, 5

Rasio F. A., Tout C. A., Lubow S. H., Livio M., 1996, ApJ, 470, 1187

Rieutord M., 2014, Fluid Dynamics: An Introduction. Springer, Berlin

Samadi R., Goupil M.-J., Lebreton Y., 2001, A\&A, 370, 147

Schaeffer N., 2013, Geochem. Geophys. Geosyst., 14, 751

Spiegel E. A., 1971, ARA\&A, 9, 323

Terquem C., Papaloizou J., Nelson R., Lin D., 1998, ApJ, 502, 788

Triaud A. H. M. J., et al., 2017, A\&A, 608, A129

Van Eylen V., Winn J. N., Albrecht S., 2016, ApJ, 824, 15
Verbunt F., Phinney E. S., 1995, A\&A, 296, 709

Vick M., Lai D., 2020, MNRAS, 496, 3767

Vidal J., Barker A. J., 2020, ApJ, 888, L31

Vidal J., Cébron D., 2017, J. Fluid Mech., 833, 469

Vidal J., Schaeffer N., 2015, Geophys. J. Int., 202, 2182

Vidal J., Cébron D., Schaeffer N., Hollerbach R., 2018, MNRAS, 475, 4579

Vidal J., Cébron D., ud Doula A., Alecian E., 2019, A\&A, 629, A142

Zahn J.-P., 1966, Ann. Astrophys, 29, 489

Zahn J.-P., 1989, A\&A, 220, 112

Zahn J.-P., 2008, EAS Publications Series, 29, 67

Zahn J.-P., Bouchet L., 1989, A\&A, 223, 112

von Boetticher A., et al., 2019, A\&A, 625, A150

\section{APPENDIX A: MESA CODE PARAMETERS}

We use MESA version 12778 (Paxton et al. 2011, 2013, 2015, 2018, 2019). The inlist file that we use is given below. We alter initial_mass to generate a given stellar model.

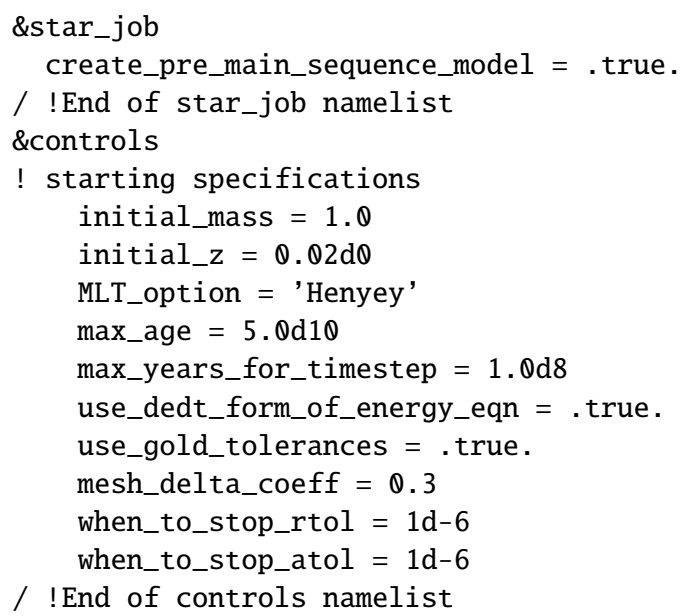

This paper has been typeset from a $\mathrm{T}_{\mathrm{E}} \mathrm{X} / \mathrm{LAT}_{\mathrm{E}} \mathrm{X}$ file prepared by the author. 